\title{
ABUNDANCES OF C, N, Sr, AND Ba ON THE RED GIANT BRANCH OF $\omega$ CENTAURI*
}

\author{
Laura M. Stanford ${ }^{1,2}$, G. S. Da COSTA ${ }^{2}$, AND John E. Norris ${ }^{2}$ \\ ${ }^{1}$ McDonald Observatory, The University of Texas at Austin, 1 University Station, C1400, Austin, TX 78712-0259, USA \\ ${ }^{2}$ Research School of Astronomy and Astrophysics, Australian National University, Weston, ACT 2611, Australia; \\ laura@astro.as.utexas.edu,gdc@mso.anu.edu.au,jen@mso.anu.edu.au \\ Received 2009 October 5; accepted 2010 March 19; published 2010 April 15
}

\begin{abstract}
Abundances relative to iron for carbon, nitrogen, strontium, and barium are presented for 33 stars on the red giant branch $(\mathrm{RGB})$ of the globular cluster $\omega$ Centauri. They are based on intermediate-resolution spectroscopic data covering the blue spectral region analyzed using spectrum synthesis techniques. The data reveal the existence of a broad range in the abundances of these elements, and a comparison with similar data for main-sequence stars enables insight into the evolutionary history of the cluster. The majority of the RGB stars were found to be depleted in carbon, i.e., $[\mathrm{C} / \mathrm{Fe}]<0$, while $[\mathrm{N} / \mathrm{Fe}]$ for the same stars shows a range of $\sim 1$ dex, from $[\mathrm{N} / \mathrm{Fe}] \approx 0.7$ to $1.7 \mathrm{dex}$. The strontium-to-iron abundance ratios varied from solar to mildly enhanced $(0.0 \leqslant[\mathrm{Sr} / \mathrm{Fe}] \leqslant 0.8)$, with $[\mathrm{Ba} / \mathrm{Fe}]$ generally equal to or greater than $[\mathrm{Sr} / \mathrm{Fe}]$. The carbon and nitrogen abundance ratios for the one known $\mathrm{CH}$ star in the sample, ROA 279, are $[\mathrm{C} / \mathrm{Fe}]=0.6$ and $[\mathrm{N} / \mathrm{Fe}]=0.5$ dex. Evidence for evolutionary mixing on the RGB is found from the fact that the relative carbon abundances on the main sequence are generally higher than those on the RGB. However, comparison of the RGB and main-sequence samples shows that the upper level of nitrogen enhancement is similar in both sets at $[\mathrm{N} / \mathrm{Fe}] \approx 2.0$ dex. This is most likely the result of primordial rather than evolutionary mixing processes. One RGB star, ROA 276, was found to have $\mathrm{Sr}$ and $\mathrm{Ba}$ abundance ratios similar to the anomalous Sr-rich main-sequence star S2015448. High-resolution spectra of ROA 276 were obtained with the Magellan Telescope/ MIKE spectrograph combination to confirm this result, revealing that ROA 276 is indeed an unusual star. For this star, calculations of the depletion effect, the potential change in surface abundance that results from the increased depth of the convective envelope as a star moves from the main sequence to the RGB, strongly suggest that the observed Sr enhancement in ROA 276 is of primordial origin, rather than originating from a surface accretion event.
\end{abstract}

Key words: globular clusters: general - globular clusters: individual ( $\omega$ Centauri)

Online-only material: color figure

\section{INTRODUCTION}

The unusual variation in abundances of stars within the globular cluster $\omega$ Centauri has been well studied over the last four decades. From the original photographic and photoelectric work of Woolley et al. (1966) and Cannon \& Stobie (1973), respectively, and the more recent analysis of CCD photometry by Sollima et al. (2005), the spread in color on the red giant branch (RGB) is apparent with distinct branches visible in the color-magnitude diagram (CMD). The large range in metallicity of over 1 dex has been studied extensively on the RGB (Norris et al. 1996, hereafter NFM96; Suntzeff \& Kraft 1996; Lee et al. 1999; Pancino et al. 2000; Rey et al. 2004; Sollima et al. 2005; Johnson et al. 2008; Calamida et al. 2009), and to a limited extent on the main-sequence and turnoff (MSTO) area (Piotto et al. 2005; Sollima et al. 2005; Stanford et al. 2006a; Kayser et al. 2006; Villanova et al. 2007).

Large ranges in abundance for all elements studied in the cluster have been found on the RGB (Norris \& Da Costa 1995a, hereafter ND95; Smith et al. 1995, 2000; Johnson et al. 2008; Calamida et al. 2009). This contrasts with what is found in normal globular clusters where typically small ranges for the light elements $(\mathrm{C}, \mathrm{N}, \mathrm{O}, \mathrm{Mg}, \mathrm{Al}$, and $\mathrm{Na}$ ) are seen. The light elements in $\omega$ Cen stars show large variations for a given $[\mathrm{Fe} / \mathrm{H}]$ (Persson et al. 1980; Brown \& Wallerstein 1993, hereafter BW93; ND95). The $\alpha$-elemental (Mg, Si, Ca, and Ti) abundance ratios with respect to iron are largely constant for

\footnotetext{
* This paper includes data gathered with the $6.5 \mathrm{~m}$ Magellan Telescopes located at Las Campanas Observatory, Chile.
}

$[\mathrm{Fe} / \mathrm{H}]<-1.0$ (BW93; Smith et al. 1995, 2000; ND95) but $[\alpha / \mathrm{Fe}]$ then decreases at metallicities greater than -1.0 (Pancino et al. 2002). Sodium ([Na/Fe]) and aluminum ([Al/Fe]) abundances are correlated, and both are anticorrelated with oxygen ([O/Fe]; BW93; ND95; Norris \& Da Costa 1995b; Smith et al. 2000). For lower metallicities (below $[\mathrm{Fe} / \mathrm{H}]<-0.8$ ), Smith et al. (2000) and Cunha et al. (2002) report constant $[\mathrm{Cu} / \mathrm{Fe}]$. Above a metallicity of $[\mathrm{Fe} / \mathrm{H}]=-1.2$, however, Pancino et al. (2002) find an increase in $[\mathrm{Cu} / \mathrm{Fe}]$ as the metallicity increases. Variations in abundance for the neutron-capture elements have also been found. The $s$-process element abundance ratios, $[s / \mathrm{Fe}]$, increase with increasing $[\mathrm{Fe} / \mathrm{H}]$, but then are constant above metallicities greater than -1.2 (ND95; Smith et al. 1995). The source of these abundance variations can be attributed to several different possibilities. The decrease in $\alpha$-elemental abundances at higher metallicities $([\mathrm{Fe} / \mathrm{H}] \geqslant-1.0)$ can be attributed to Type Ia supernovae enrichment. The origin of the $s$-process enrichment is possibly due to ejecta from asymptotic giant branch (AGB) stars, and the iron peak and metal abundance patterns are consistent with primordial enrichment from Type II supernovae.

At least three different processes are likely to be involved. The first are the processes that mix material within the stars themselves. These include the first dredge up on the RGB in which the surface layers of the star are mixed with lower processed material (Iben 1965). This can, in principle, account for some of the variations seen within the RGB stars of $\mathrm{C}, \mathrm{N}$, and $\mathrm{O}$. These processes, however, are unlikely to account for the variations seen in elements heavier than oxygen. 
Table 1

List of RGB Objects with Observing Dates and Grating Used

\begin{tabular}{ccc}
\hline \hline $\begin{array}{c}\text { Grating } \\
(1)\end{array}$ & $\begin{array}{c}\text { Date } \\
(2)\end{array}$ & $\begin{array}{c}\text { Star ID } \\
(3)\end{array}$ \\
\hline $600 \mathrm{I}$ & 2005 May & ROA: $24,40,43,46,53,58,84,132,150,159$ \\
& & ROA: $162,171,179,219,248,252,253,371$ \\
$1200 \mathrm{I}$ & ROA: $40,42,43,46,48,55,65,74,84,94139$ \\
& $2002 \mathrm{Feb} / \mathrm{Jun}$ & ROA: $159,179,201,213,219,253,276,279,300$ \\
& & ROA: $324,336,357,425,447,477,480,517$, \\
& & OC: $140419,222068,230189,242056,250606,263340$ \\
& OC: $305654,312058,321293,618774,801639$
\end{tabular}

Second, an enriched object may have accreted matter onto its surface layers from either stellar winds from AGB stars or interstellar material. As the accretion events only affect the surface layers of the star, once it ascends the RGB and the convective envelope deepens, the accreted matter will be mixed with the internal layers, thereby reducing the level of enrichment. A comparison of MS and RGB stars may then show higher abundances for the unevolved MS stars compared to those on the RGB. Johnson et al. (2009) suggest large binary fractions, and hence accretion of enhanced material, as the source of $25 \%$ of their sample with $[\mathrm{La} / \mathrm{Eu}] \geqslant 1$. Calamida et al. (2009) also suggest the same scenario to explain the $\sim 20 \%$ of $\mathrm{CN}$-strong RGB stars identified in their sample. However, this is in contrast to that observed by Mayor et al. (1996) who monitored $~ 310$ giants in $\omega$ Cen over a ten-year period and found the binary fraction to be as low as 3\%-4\%.

The third possibility is that the variations have a primordial origin; i.e., the stars formed from gas enriched by a combination of AGB stars, massive stars, and/or supernovae. It is likely that more than one of these scenarios is at work.

Nevertheless, a comparison between abundances obtained from MS and RGB stars may help to distinguish among the various processes involved in the enrichment of this unusual star cluster. In particular, in the absence of thermohaline mixing (discussed further in Section 5), it can distinguish whether enrichment of $s$-process and CNO elements is due to surface contamination (which would be diluted by the growing convective envelope as the stars move on to the giant branch) or is instead uniform throughout the stars. An abundance analysis of MSTO stars has already been performed by the present authors (Stanford et al. 2007) for carbon, nitrogen, and strontium. In this paper, we investigate abundances on the RGB in a similar manner. The sample of RGB stars and their observations is described Section 2. In Section 3, we discuss the stellar parameters and the techniques used to obtain abundances for $\mathrm{C}, \mathrm{N}, \mathrm{Sr}$, and $\mathrm{Ba}$ in the stars. The results are presented in Section 4. In Section 5, we present comparisons between the RGB and MSTO and discuss the consequences.

\section{THE RED GIANT BRANCH SAMPLE AND OBSERVATIONS}

In order to compare abundances found on the main sequence (MS; Stanford et al. 2007) with those on the RGB, spectra were obtained for a sample of RGB stars. These objects have $-2.6<M_{\mathrm{v}}<-0.28$, and are among the brightest RGB stars in the cluster. All lie above the luminosity where the first dredge up occurs and most, if not all, have luminosities that place them above the bump in the RGB luminosity function. The objects selected for observation covered a range in metallicities and several stars were included due to the known nature of their $\mathrm{CH}$ feature, or high $s$-process abundances. These objects are more likely to have higher relative abundances and to be useful for comparisons with the unusual stars on the MS. While most of these objects are ROA objects (Woolley et al. 1966), 11 come from the list of Pancino et al. (2002).

The spectra of the RGB stars were obtained on the ANU's 2.3 $\mathrm{m}$ telescope at Siding Spring Observatory. Data were obtained in 2002 February, 2002 June, and 2003 May with the Double Beam Spectrograph using 600 and 1200 line $\mathrm{mm}^{-1}$ gratings. The stars observed are listed in Table 1 . Spectra for eighteen RGB stars were obtained with the 600 line $\mathrm{mm}^{-1}$ grating. The 1200 I grating was used to observe 39 RGB stars, 8 of which were in common with those observed with the lower resolution grating. The low-resolution (R600) data covered a wavelength range $3500-5400 \AA$ and had a resolution of $2.2 \AA$ FWHM. The high-resolution (R1200) spectra covered a wavelength range of 3700-4600 $⿱$ and their resolution was $1.2 \AA$ FWHM. Reduction and wavelength calibration was performed using standard routines in IRAF and Figaro.

In addition to the above medium-resolution spectra, highresolution data were also obtained for one outstanding object in the sample, ROA 276, together with two other comparison objects, ROA 46 and ROA 150. These spectra were obtained with the Magellan Telescope/MIKE spectrograph combination during 2007 June $22-23$ using a 0.5 arcsec slit, yielding resolving power 47,000 , and resolution $0.12 \AA \mathrm{FWHM}$. This material will be discussed in Section 4.4.3.

\section{STELLAR PARAMETERS}

Stellar parameters for our sample were obtained primarily from the literature. ND95 listed temperatures $\left(T_{\text {eff }}\right)$, gravities $(\log g)$, metallicities $([\mathrm{Fe} / \mathrm{H}])$, and microturbulent velocities $\left(v_{\mathrm{t}}\right)$ for a large fraction of the RGB objects for which we have data, and these were used for consistency between objects. Temperatures and gravities were also obtained from Persson et al. (1980) for objects that were not in ND95. Stellar parameters for several RGB stars were also obtained from Pancino (2003, hereafter PAN03) which are identified by the "OC" prefix. For objects that did not have metallicities listed in ND95, $[\mathrm{Fe} / \mathrm{H}]$ was determined using $[\mathrm{Ca} / \mathrm{H}]$ from NFM96 where $[\mathrm{Fe} / \mathrm{H}]=[\mathrm{Ca} / \mathrm{H}]-[\mathrm{Ca} / \mathrm{Fe}]$. Following Smith et al. (1995) and Pancino et al. (2000), we adopt the following $[\mathrm{Ca} / \mathrm{Fe}]$ relation:

$$
[\mathrm{Ca} / \mathrm{Fe}]= \begin{cases}0.3 & \text { for }[\mathrm{Fe} / \mathrm{H}] \leqslant-1.0 \\ -0.3 \times[\mathrm{Fe} / \mathrm{H}] & \text { for }-1.0<[\mathrm{Fe} / \mathrm{H}] \leqslant 0.0 .\end{cases}
$$

A small number of objects in our sample had no stellar parameter information from the sources listed above, or from others in 
Table 2

Stellar Parameters for RGB Stars in $\omega$ Cen

\begin{tabular}{|c|c|c|c|c|c|c|c|}
\hline ID & $V^{\mathrm{a}}$ & $B-V^{\mathrm{b}}$ & $T_{\text {eff }}$ & $\log g$ & {$[\mathrm{Fe} / \mathrm{H}]$} & $V_{t}$ & Source $^{\mathrm{c}}$ \\
\hline ROA 40 & 11.37 & 1.48 & 4200 & 0.5 & -1.69 & 2.3 & 1 \\
\hline ROA 42 & 11.64 & 1.49 & 4150 & 0.5 & -1.69 & 2.0 & 1 \\
\hline ROA 43 & 11.62 & 1.62 & 3950 & 0.4 & -1.47 & 2.1 & 1 \\
\hline ROA 46 & 11.54 & 1.55 & 4050 & 0.5 & -1.67 & 2.2 & 1 \\
\hline ROA 48 & 11.51 & 1.58 & 4050 & 0.5 & -1.76 & 2.5 & 1 \\
\hline ROA 53 & 11.58 & 1.64 & 3950 & 0.4 & -1.67 & 2.3 & 1 \\
\hline ROA 58 & 11.67 & 1.43 & 4200 & 0.6 & -1.73 & 2.2 & 1 \\
\hline ROA 65 & 11.61 & 1.50 & 4050 & 0.6 & -1.72 & 2.1 & 1 \\
\hline ROA 74 & 11.78 & 1.37 & 4250 & 0.7 & -1.80 & 2.2 & 1 \\
\hline ROA 84 & 11.87 & 1.66 & 3900 & 0.5 & -1.36 & 1.9 & 1 \\
\hline ROA 94 & 11.80 & 1.39 & 4200 & 0.7 & -1.78 & 2.1 & 1 \\
\hline ROA 132 & 12.05 & 0.87 & 3900 & 0.3 & -1.37 & 2.2 & 1 \\
\hline ROA 139 & 11.98 & 1.45 & 4150 & 0.8 & -1.46 & 1.6 & 1 \\
\hline ROA 150 & 12.00 & 1.69 & 3950 & 0.6 & -1.25 & 2.2 & 1 \\
\hline ROA 159 & 12.04 & 1.34 & 4300 & 0.9 & -1.72 & 2.0 & 1 \\
\hline ROA 162 & 12.14 & $\ldots$ & 3950 & 0.7 & -1.10 & 2.1 & 1 \\
\hline ROA 171 & 12.07 & 1.47 & 4100 & 0.7 & -1.43 & 1.9 & 1 \\
\hline ROA 179 & 12.21 & 1.65 & 3850 & 0.5 & -1.10 & 1.5 & 1 \\
\hline ROA 213 & 12.22 & 1.12 & 4500 & 1.1 & -1.83 & 1.9 & 1 \\
\hline ROA 219 & 12.20 & $\ldots$ & 4000 & 0.7 & -1.10 & 2.2 & 1 \\
\hline ROA 248 & 12.43 & 1.70 & 3850 & 0.6 & -0.78 & 1.6 & 1 \\
\hline ROA 252 & 12.30 & 1.20 & 4400 & 1.1 & -1.74 & 2.0 & 1 \\
\hline ROA 253 & 12.34 & 1.37 & 4300 & 1.0 & -1.39 & 1.9 & 1 \\
\hline ROA 276 & 12.37 & 1.38 & 4000 & 0.7 & -0.57 & 2.0 & 3,5 \\
\hline ROA 279 & 12.32 & $\ldots$ & 4350 & 1.1 & -1.69 & 2.0 & 1 \\
\hline ROA 300 & 12.71 & 1.68 & 3900 & 0.7 & -0.77 & 2.0 & 2,3 \\
\hline ROA 324 & 12.59 & 1.54 & 4000 & 0.7 & -0.39 & 1.9 & 3,5 \\
\hline ROA 357 & 12.69 & 1.43 & 4000 & 0.8 & -0.85 & 1.8 & 1 \\
\hline ROA 371 & 12.68 & 1.61 & 4000 & 0.9 & -0.79 & 1.6 & 1 \\
\hline ROA 425 & 12.73 & $\ldots$ & 3650 & 0.6 & -0.32 & 2.0 & 2,3 \\
\hline ROA 447 & 12.80 & 1.64 & 3700 & 0.7 & -0.18 & 2.0 & 2,3 \\
\hline ROA 480 & 12.98 & 1.25 & 4350 & 1.3 & -0.95 & 1.8 & 1 \\
\hline OC 140419 & 13.49 & 1.22 & 4200 & 1.5 & -0.65 & 2.0 & 4 \\
\hline OC 263340 & 13.62 & 1.13 & 4400 & 1.9 & -0.63 & 1.2 & 4 \\
\hline OC 305654 & 13.38 & 1.29 & 4200 & 1.6 & -0.65 & 1.4 & 4 \\
\hline OC 321293 & 13.69 & 1.15 & 4300 & 1.6 & -0.71 & 1.4 & 4 \\
\hline
\end{tabular}

Notes.

a NFM96 and references therein; Pancino (2003).

b van Leeuwen (2000) and Pancino (2003).

c Source: (1) ND95; (2) PFCAM; (3) NFM96; (4) Pancino 2003; (5) this study.

the literature. In these cases a metallicity was determined from $[\mathrm{Ca} / \mathrm{H}]$, of NFM96, as described above. This metallicity was then used to find other RGB stars in our sample with similar values $(\Delta[\mathrm{Fe} / \mathrm{H}]= \pm 0.2)$ for which we had stellar parameters. A least-squares fit was then performed in $V-\log g$ space for the chosen comparison stars to obtain a gravity for the star from its $V$ magnitude. Similarly, a temperature was obtained from a least-squares fit in $(B-V)-T_{\text {eff }}$ space. As most RGB stars have a microturbulent velocity of $\sim 2.0 \mathrm{~km} \mathrm{~s}^{-1}$, this value was assumed for these objects. However, there remained a small number of stars for which we were unable to obtain stellar parameters (ROA 55, 577; and OC 230189, 242056, 250606, 312058, 801639) and consequently these stars were not included in the analysis.

Table 2 lists the stellar parameters used for each star, with ROA or OC number in Column $1, V$ magnitude, and $B-V$ color in Columns 2 and 3 , and $T_{\text {eff }}, \log g$, metallicity, and microturbulence in Columns 4-7, respectively. The sources of the stellar parameters are listed against each star for clarification in Column 8.

Abundances of $[\mathrm{O} / \mathrm{Fe}]$ and $[\mathrm{Ca} / \mathrm{Fe}]$ were obtained from ND95 and PAN03. In the instances where these were not available, the abundance ratios of these elements followed Equation (1) above.

Two objects, ROA 336 and 517, observed with the higher resolution grating, had high metallicities $([\mathrm{Ca} / \mathrm{H}]=-0.33$ and 0.02, respectively; Norris et al. 1996). Spectrum synthesis was attempted but it was found that for these cool, metalrich stars the models do not produce an adequate fit to the observed spectra. Consequently, these objects were not analyzed further. The remaining sample then consisted of 23 objects with high-resolution (1.2 $\AA$ ) and 16 stars with low-resolution $(2.2 \AA)$ spectra, with 7 stars in common.

\section{ABUNDANCES}

The abundance analysis used spectrum synthesis techniques for each of the objects, adopting the stellar parameters listed in Table 2. Kurucz (1993) stellar models were employed with atomic line lists from R. A. Bell (2000, private communication) and Kurucz molecular line lists. As with our study of abundances on the MS/subgiant branch (Stanford et al. 2007) the synthesis code developed by Cottrell (Cottrell \& Norris 1978) was used to generate synthetic spectra. These were broadened to $1.1 \AA$ and $2.2 \AA$ to match the observed higher and lower resolution data, respectively. The solar abundances and gf values for $\mathrm{CH}, \mathrm{CN}$, Sr II $4077 \AA$, Sr II $4215 \AA$, and Ba II $4554 \AA$ used in Stanford et al. (2007) were again employed.

As for the MSTO data, the $\mathrm{CH}$ feature at $4300 \AA$ was analyzed first. The resulting $[\mathrm{C} / \mathrm{Fe}]$ was then adopted in the analysis of the $\mathrm{CN}$ features. Due to the cooler nature of the RGB stars in comparison with the MSTO stars, the violet $\mathrm{CN}$ at $3883 \AA$ is often saturated making any abundance determination from this band uncertain. Therefore, the blue CN band at $4216 \AA$ was used for the RGB stars. The effect this has on the derived abundances is minimal, and was tested using an RGB object (ROA 40) with low $\mathrm{N}$ enhancement $([\mathrm{N} / \mathrm{Fe}]=0.6)$. For this star, the violet $\mathrm{CN}$ band was not saturated and consistent results were found for $[\mathrm{N} / \mathrm{Fe}]$ from both bands.

Finally, the SrII $4077 \AA$, Sr II $4215 \AA$, and Ba II $4554 \AA$ features were analyzed. In contrast to the MSTO data, Ba abundances were able to be determined for each RGB star. This was due to the higher resolution and signal-to-noise ratio of the data and/or cooler temperatures. The Sr II $4215 \AA$ line was not analyzed if $[\mathrm{N} / \mathrm{Fe}]>0.5$ owing to contamination from the $4215 \AA \mathrm{CN}$ features. In the synthetic spectra, several lines (e.g., iron, titanium, samarium, $\mathrm{CH}$ ) were present near the $\mathrm{Sr}$ II $4077 \AA$ feature $(\lambda \lambda 4075-4077)$ that were more clearly separated in the observed high-resolution (R1200) spectra. In the lower resolution data (R600), these features were blended with the Sr II line making the abundance determined from the R600 data slightly more uncertain. The $\mathrm{Sr}$ abundances were determined from the models both with and without these lines, which led to the conclusion that they did not significantly affect the $[\mathrm{Sr} / \mathrm{Fe}]$ measurements. This was particularly the case when the $\mathrm{Sr}$ feature was strong.

The effect of hyperfine splitting (hfs) on the derived abundance from the Ba II $4554 \AA$ line was investigated following Norris et al. (1997). Two line lists were used: one that included hfs and isotopic components for Ba II $4554 \AA$, and one that did not. A comparison of abundances was made for a series of stellar parameters and $[\mathrm{Ba} / \mathrm{Fe}]$ spanning the same range as for the RGB sample. Little difference $(\Delta[\mathrm{Ba} / \mathrm{Fe}]<0.05)$ was found between the abundances obtained from the spectra with and without the inclusion of isotopes and hfs. 
Table 3

Abundances for Carbon, Nitrogen, Strontium, and Barium, and the Individual Errors for the R600 Data

\begin{tabular}{|c|c|c|c|c|c|c|c|c|c|}
\hline \multirow[t]{2}{*}{ ID } & \multirow[t]{2}{*}[\mathrm{Fe}/\mathrm{H}]{} & \multicolumn{2}{|c|}{ Carbon } & \multicolumn{2}{|c|}{ Nitrogen } & \multicolumn{2}{|c|}{ Strontium } & \multicolumn{2}{|c|}{ Barium } \\
\hline & & $\mathrm{ab}^{\mathrm{a}}$ & $\mathrm{e}^{\mathrm{a}}$ & $\mathrm{ab}^{\mathrm{a}}$ & $\mathrm{e}^{\mathrm{a}}$ & $\mathrm{ab}^{\mathrm{a}}$ & $\mathrm{e}^{\mathrm{a}}$ & $\mathrm{ab}^{\mathrm{a}}$ & $\mathrm{e}^{\mathrm{a}}$ \\
\hline ROA 40 & -1.69 & -0.20 & 0.24 & 0.6 & 0.31 & 0.3 & 0.36 & 0.5 & 0.32 \\
\hline ROA 43 & -1.47 & -0.30 & 0.24 & 1.2 & 0.38 & 0.4 & 0.36 & 0.5 & 0.18 \\
\hline ROA 46 & -1.67 & -0.70 & 0.24 & 0.5 & 0.38 & 0.0 & 0.44 & 0.3 & 0.32 \\
\hline ROA 53 & -1.67 & -0.40 & 0.29 & 0.7 & 0.38 & -0.1 & 0.44 & 0.4 & 0.32 \\
\hline ROA 58 & -1.73 & -0.80 & 0.29 & 0.6 & 0.31 & -0.1 & 0.36 & 0.1 & 0.32 \\
\hline ROA 84 & -1.36 & -0.10 & 0.24 & 0.9 & 0.35 & 0.7 & 0.28 & 0.9 & 0.18 \\
\hline ROA 132 & -1.37 & -0.50 & 0.29 & 0.9 & 0.35 & 0.0 & 0.31 & 0.4 & 0.22 \\
\hline ROA 150 & -1.25 & -1.00 & 0.24 & 1.5 & 0.38 & 0.6 & 0.28 & 0.7 & 0.18 \\
\hline ROA 159 & -1.72 & -0.50 & 0.24 & 0.2 & 0.38 & -0.2 & 0.44 & -0.2 & 0.41 \\
\hline ROA 162 & -1.10 & -0.60 & 0.24 & 1.5 & 0.38 & 0.3 & 0.36 & 0.8 & 0.22 \\
\hline ROA 171 & -1.43 & -0.20 & 0.29 & 0.9 & 0.31 & 0.7 & 0.28 & 0.6 & 0.22 \\
\hline ROA 179 & -1.10 & 0.10 & 0.24 & $\ldots$ & 0.35 & 0.5 & 0.28 & 0.8 & 0.18 \\
\hline ROA 219 & -1.10 & 0.00 & 0.24 & 0.5 & 0.31 & 0.4 & 0.28 & 0.7 & 0.18 \\
\hline ROA 248 & -0.78 & -1.00 & 0.29 & 1.8 & 0.38 & 0.6 & 0.31 & 0.6 & 0.18 \\
\hline ROA 253 & -1.39 & -0.70 & 0.24 & 1.4 & 0.31 & 0.4 & 0.36 & 0.8 & 0.32 \\
\hline ROA 371 & -0.79 & -0.90 & 0.29 & 1.4 & 0.35 & 0.4 & 0.31 & 0.9 & 0.27 \\
\hline
\end{tabular}

Note. ${ }^{\mathrm{a}} \mathrm{ab}=[\mathrm{X} / \mathrm{Fe}] ; \mathrm{e}=\Delta[\mathrm{X} / \mathrm{Fe}]$.

\subsection{Error Analysis}

An error analysis was performed by varying the temperature, gravity, metallicity, and microturbulence, and redetermining the abundances for several stars in both the R600 and R1200 samples. The parameters were varied by $\Delta T= \pm 100 \mathrm{~K}$, $\Delta \log g= \pm 0.2, \Delta[\mathrm{Fe} / \mathrm{H}]= \pm 0.2$, and $\Delta v_{\mathrm{t}}= \pm 0.3$.

The derived carbon abundance is dependent on the $\mathrm{O}$ abundance, which was taken from ND95 and PAN03 when available. The oxygen abundance $[\mathrm{O} / \mathrm{Fe}]$ was varied by \pm 0.2 and the spectrum synthesis was repeated to determine the error in $[\mathrm{C} / \mathrm{Fe}]$ due to the possible uncertainty in the oxygen abundance. This error was then included in the final error of $[\mathrm{C} / \mathrm{Fe}]$. These individual errors were added in quadrature and gave a final error $\Delta[\mathrm{C} / \mathrm{Fe}]_{\mathrm{R} 600}=0.26$ for the $\mathrm{R} 600$ data and $\Delta[\mathrm{C} / \mathrm{Fe}]_{\mathrm{R} 1200}=0.22$ for the R1200. As the nitrogen abundance determination was dependent on the adopted $[\mathrm{C} / \mathrm{Fe}]$, the final $[\mathrm{N} / \mathrm{Fe}]$ error also included the uncertainty in $[\mathrm{C} / \mathrm{Fe}]$. The errors in the $\mathrm{N}$ abundance were $\Delta[\mathrm{N} / \mathrm{Fe}]_{\mathrm{R} 600}=0.35$ and $\Delta[\mathrm{N} / \mathrm{Fe}]_{\mathrm{R} 1200}=0.31$ for the R600 and R1200 data, respectively. The uncertainties in Sr and $\mathrm{Ba}$ abundances were determined to be $\Delta[\mathrm{Sr} / \mathrm{Fe}]_{\mathrm{R} 600}=0.37$ and $\Delta[\mathrm{Sr} / \mathrm{Fe}]_{\mathrm{R} 1200}=0.35$, and $\Delta[\mathrm{Ba} / \mathrm{Fe}]_{\mathrm{R} 600}=0.31$ and $\Delta[\mathrm{Ba} / \mathrm{Fe}]_{\mathrm{R} 1200}=0.28$.

In addition to the stellar parameters and oxygen abundance errors, a measurement uncertainty exists in the determination of the individual abundances. This was estimated by visual inspection of the quality of the fit for each star for carbon, nitrogen, strontium, and barium, and combined with the general errors above. These uncertainties are listed for each star individually in Table 3 for the low-resolution data and Table 4 for the R1200 ones.

\subsection{Abundances of $C, N, S r$, and $B a$}

The low-resolution (R600) abundance results are shown in Table 3. For all but one star in this data set, the $\mathrm{C}$ abundance was found to be depleted $([\mathrm{C} / \mathrm{Fe}]<0.0)$. The object with the highest $\mathrm{C}$ abundance was the CO-strong star ROA 179 with $[\mathrm{C} / \mathrm{Fe}]=0.1$. Large overabundances in nitrogen were seen in $\mathrm{C}$-depleted objects, and moderate enhancements of $\mathrm{Sr}$ and $\mathrm{Ba}$ were found. In general, the Ba enhancements were greater than or equal to those for $\mathrm{Sr}$, with $[\mathrm{Ba} / \mathrm{Sr}] \geqslant 0.0$. Figure 1 shows an example of low-resolution spectra of the CO-strong star ROA 219 with the spectrum synthesis results.

Similar patterns to those found in the R600 data are also seen in the R1200 data (Table 4). Most stars are C depleted and enhanced in nitrogen. $\mathrm{Sr}$ and $\mathrm{Ba}$ abundances again show moderate enhancements with $[\mathrm{Ba} / \mathrm{Sr}] \geqslant 0.0$ for the majority of stars.

The six stars for which both R600 and R1200 data were obtained (see Figure 2) show mean abundance differences (with the standard error of the mean), $\Delta(\mathrm{R} 600-\mathrm{R} 1200)=$ $0.08 \pm 0.05$ for $[\mathrm{C} / \mathrm{Fe}], 0.10 \pm 0.06$ for $[\mathrm{N} / \mathrm{Fe}], 0.18 \pm 0.07$ for $[\mathrm{Sr} / \mathrm{Fe}]$, and $0.17 \pm 0.09$ for $[\mathrm{Ba} / \mathrm{Fe}]$. We will need to keep in mind the possibility of a systematic offset between the R600 and R1200 [Sr/Fe] values in the subsequent discussion.

\subsection{Comparisons with Other Studies}

The abundances determined here can be compared with those in the literature for the same stars. ND95 determined $[\mathrm{C} / \mathrm{Fe}]$ from the $\mathrm{G}$ band, $[\mathrm{N} / \mathrm{Fe}]$ from the blue $\mathrm{CN}$ band, and $[\mathrm{Ba} / \mathrm{Fe}]$ from three lines (not including the one used here) for a large fraction of our sample. Although they did not observe $\mathrm{Sr}$ in their investigation of RGB stars, other light $s$-process elements such as yttrium and zirconium were included and these can be compared with the $\mathrm{Sr}$ abundances determined here for stars in common between the two studies. BW93 measured carbon (from the $\mathrm{G}$ band) and nitrogen (from the red $\mathrm{CN}$ band at $7950 \AA$ ) , among other elements, and have four stars in common (ROA 48, 74, 84, 213) with this study. The RGB stars with OC designations come from PAN03, and the results found here can be compared with the abundances of $\mathrm{Ba}, \mathrm{Y}$, and $\mathrm{Zr}$ published there.

Figure 3 shows the comparisons between the present study on the one hand, and ND95 and BW93, on the other, for [C/ $\mathrm{Fe}]$ and $[\mathrm{N} / \mathrm{Fe}]$. The barium abundances were compared with those found in ND95 and PAN03 for stars in common. The R600 data show good agreement for the carbon abundances with ND95 and BW93. The mean abundance differences, in the sense this study - other study, were $\langle\Delta[\mathrm{C} / \mathrm{Fe}]\rangle_{\mathrm{ND}}=$ $-0.11 \pm 0.13$ and $\langle\Delta[\mathrm{C} / \mathrm{Fe}]\rangle_{\mathrm{BW}}=-0.10$ for the one star in common. The R1200 data show a similar pattern where $\langle\Delta[\mathrm{C} / \mathrm{Fe}]\rangle_{\mathrm{ND}}=-0.11 \pm 0.17$ and $\langle\Delta[\mathrm{C} / \mathrm{Fe}]\rangle_{\mathrm{BW}}=-0.19 \pm$ 
Table 4

Abundances for Carbon, Nitrogen, Strontium, and Barium, and the Individual Errors for the R1200 Data

\begin{tabular}{|c|c|c|c|c|c|c|c|c|c|}
\hline \multirow[t]{2}{*}{ ID } & \multirow[t]{2}{*}[\mathrm{Fe}/\mathrm{H}]{} & \multicolumn{2}{|c|}{ Carbon } & \multicolumn{2}{|c|}{ Nitrogen } & \multicolumn{2}{|c|}{ Strontium } & \multicolumn{2}{|c|}{ Barium } \\
\hline & & $a b^{a}$ & $\mathrm{e}^{\mathrm{a}}$ & $a b^{a}$ & $\mathrm{e}^{\mathrm{a}}$ & $a b^{a}$ & $\mathrm{e}^{\mathrm{a}}$ & $a b^{a}$ & $\mathrm{e}^{\mathrm{a}}$ \\
\hline ROA 40 & -1.69 & -0.10 & 0.16 & 0.4 & 0.22 & -0.1 & 0.34 & 0.3 & 0.28 \\
\hline ROA 43 & -1.47 & -0.40 & 0.21 & 1.0 & 0.26 & 0.4 & 0.37 & 0.8 & 0.31 \\
\hline ROA 46 & -1.67 & -0.90 & 0.25 & $\ldots$ & 0.43 & $\ldots$ & $\ldots$ & 0.0 & 0.44 \\
\hline ROA 48 & -1.76 & -0.80 & 0.18 & 0.4 & 0.43 & -0.2 & 0.40 & -0.2 & 0.44 \\
\hline ROA 65 & -1.72 & -0.60 & 0.18 & $\ldots$ & $\ldots$ & $\ldots$ & $\ldots$ & $\ldots$ & $\ldots$ \\
\hline ROA 74 & -1.80 & -0.80 & 0.18 & 0.8 & 0.43 & 0.0 & 0.34 & 0.0 & 0.36 \\
\hline ROA 84 & -1.36 & -0.10 & 0.25 & 0.8 & 0.30 & 0.5 & 0.37 & 0.5 & 0.36 \\
\hline ROA 94 & -1.78 & -0.70 & 0.18 & 0.0 & 0.43 & 0.0 & 0.34 & 0.0 & 0.36 \\
\hline ROA 139 & -1.46 & -0.90 & 0.21 & 0.5 & 0.34 & 0.0 & 0.34 & 0.2 & 0.31 \\
\hline ROA 179 & -1.10 & 0.00 & 0.25 & $\ldots$ & 0.34 & 0.3 & 0.34 & 0.5 & 0.28 \\
\hline ROA 213 & -1.83 & -0.50 & 0.18 & 0.0 & $\ldots$ & $\ldots$ & $\ldots$ & $\ldots$ & $\ldots$ \\
\hline ROA 219 & -1.10 & -0.20 & 0.21 & 0.7 & 0.30 & 0.1 & 0.34 & 0.5 & 0.28 \\
\hline ROA 253 & -1.39 & -0.70 & 0.21 & 1.3 & 0.26 & 0.3 & 0.34 & 0.7 & 0.31 \\
\hline ROA 276 & -0.57 & -0.80 & 0.21 & 0.4 & 0.34 & 0.8 & 0.34 & 0.2 & 0.28 \\
\hline ROA 279 & -1.69 & 0.60 & 0.25 & 0.5 & 0.30 & 0.6 & 0.34 & 0.6 & 0.28 \\
\hline ROA 324 & -0.39 & -1.00 & 0.34 & 1.3 & 0.30 & 0.0 & 0.34 & 0.0 & 0.28 \\
\hline ROA 357 & -0.85 & -0.80 & 0.25 & 1.4 & 0.30 & 0.0 & 0.40 & 0.6 & 0.28 \\
\hline ROA 425 & -0.32 & $\ldots$ & $\ldots$ & $\ldots$ & $\ldots$ & 0.0 & 0.40 & 0.3 & 0.36 \\
\hline ROA 480 & -0.95 & -0.60 & 0.21 & 0.6 & 0.22 & 0.6 & 0.34 & 0.6 & 0.28 \\
\hline OC 140419 & -0.65 & -0.60 & 0.25 & 0.7 & 0.26 & 0.3 & 0.34 & 05 & 0.31 \\
\hline OC 263340 & -0.63 & -0.40 & 0.21 & 0.5 & 0.22 & 0.6 & 0.34 & 0.7 & 0.28 \\
\hline OC 305654 & -0.65 & -0.30 & 0.25 & 1.0 & 0.30 & 0.0 & 0.34 & 0.4 & 0.36 \\
\hline OC 321293 & -0.71 & 0.40 & 0.21 & 0.3 & $\ldots$ & 0.1 & 0.34 & 0.5 & 0.31 \\
\hline
\end{tabular}

Note. ${ }^{\mathrm{a}} \mathrm{ab}=[\mathrm{X} / \mathrm{Fe}] ; \mathrm{e}=\Delta[\mathrm{X} / \mathrm{Fe}]$.

0.19 . The nitrogen abundances determined from both the R600 and the bulk of the R1200 data show offsets to the ND95 study of $\langle\Delta[\mathrm{N} / \mathrm{Fe}]\rangle_{\mathrm{ND}}=0.52 \pm 0.20$ and $\langle\Delta[\mathrm{N} / \mathrm{Fe}]\rangle_{\mathrm{ND}}=0.12 \pm$ 0.19 , respectively. Similar results are found when we compare our abundances with those of BW93 with $\langle\Delta[\mathrm{N} / \mathrm{Fe}]\rangle_{\mathrm{BW}}=$ 0.15 for the one star in common in the R600 sample, and $-0.27 \pm 0.19$ for the R1200 data, respectively. The reason for the offset of $\sim 0.5 \mathrm{dex}$ in $[\mathrm{N} / \mathrm{Fe}]$ for the R600 data is probably due to the lower resolution of the data. As for $[\mathrm{Sr} / \mathrm{Fe}]$, we need to keep in mind the possibility of a systematic offset between the R600 and R1200 [N/Fe] values in the subsequent discussions.

The barium abundances obtained from the R600 data are greater by $\langle\Delta[\mathrm{Ba} / \mathrm{Fe}]\rangle_{\mathrm{ND}}=0.24 \pm 0.21$ and $\langle\Delta[\mathrm{Ba} / \mathrm{Fe}]\rangle_{\mathrm{P} 03}=$ $-0.22 \pm 0.21$ compared with other studies. This is most likely due to the low resolution of our data and the blended nature of the Ba II $4554 \AA$ feature used for the analysis. The R1200 data show better agreement with ND95 with $\langle\Delta[\mathrm{Ba} / \mathrm{Fe}]\rangle_{\mathrm{ND}}=$ $0.08 \pm 0.22$. The determination of $[\mathrm{Ba} / \mathrm{Fe}]$ is somewhat easier for these spectra due to the reduced blending of the feature. From the four stars in common with PAN03, the barium abundances determined here for the R1200 data are lower by $\langle\Delta[\mathrm{Ba} / \mathrm{Fe}]\rangle_{\mathrm{P} 03}=-0.69 \pm 0.18$. As the same stellar parameters were used in both studies, the difference in abundance is most likely due to the differing nature of the analysis. Spectrum synthesis techniques were employed here for the Ba II $4554 \AA$ line, while PAN03 used an equivalent width analysis and different lines.

Figure 4 shows the comparison between the $\mathrm{Sr}$ abundances obtained here compared with $[\mathrm{Y} / \mathrm{Fe}]$ and $[\mathrm{Zr} / \mathrm{Fe}]$ obtained by ND95 and PAN03. The lower panels show the averaged $[\mathrm{Y} / \mathrm{Fe}]$ and $[\mathrm{Zr} / \mathrm{Fe}]$ abundances designated here as $[\mathrm{ls} / \mathrm{Fe}]$. The mean differences between the (R600 data) - (other studies) are $\langle\Delta[1 \mathrm{~s} / \mathrm{Fe}]\rangle_{\mathrm{ND}}=0.14 \pm 0.17$ and $\langle\Delta[\mathrm{ls} / \mathrm{Fe}]\rangle_{\mathrm{P} 03}=-0.11 \pm$
0.17 . The R1200 data show similar abundance patterns for $\mathrm{Sr}$ compared with the other light elements studied in ND95, with $\langle\Delta[\mathrm{ls} / \mathrm{Fe}]\rangle_{\mathrm{ND}}=0.20 \pm 0.30$. The comparisons of the light $s$-process elements with PAN03 show $\langle\Delta[\mathrm{ls} / \mathrm{Fe}]\rangle_{\mathrm{P} 03}=$ $-0.32 \pm 0.25$ similar to that found for the barium abundances.

\subsection{Results}

\subsubsection{Carbon and Nitrogen}

The carbon and nitrogen abundances for the R600 (gray points) and R1200 (black points) resolution data are shown as functions of metallicity in Figure 5. Objects in common between the R600 and R1200 data sets are joined by solid lines. The abundances have been plotted according to whether they are CO-strong (open circles) or CO-weak (closed circles) following the convention of ND95. Crosses are plotted for which there is no information regarding their $\mathrm{CO}$ status. In general, a CO-strong star is found to have high $[\mathrm{C} / \mathrm{Fe}]$ and relatively low $[\mathrm{N} / \mathrm{Fe}]$. Conversely, a CO-weak star shows low carbon abundances and high nitrogen ones.

Figure 6 plots the carbon abundances against those of nitrogen for the R600 (gray dots) and R1200 (black dots) resolution data. Again, objects in common between the two data sets are joined by solid lines. An anticorrelation can be seen within the data, but there is no evidence for bimodality. A least-squares fit was performed on the data, indicated by the solid line. The $p$-value for the least-squares fit was 0.183 , indicating some correlation but only a very moderate association between the carbon and nitrogen abundances. ${ }^{2}$

\footnotetext{
2 The $p$-value for the least-squares fit indicates the probability that the sample was drawn from the population being tested given the assumption that the null hypothesis is true. A p-value of 1 gives a $100 \%$ probability of the null hypothesis being true, while a $p$-value $=0.05$ gives a $5 \%$ probability. In this case, the null hypothesis was that the $[\mathrm{C} / \mathrm{Fe}]$ and $[\mathrm{N} / \mathrm{Fe}]$ abundances were unrelated.
} 

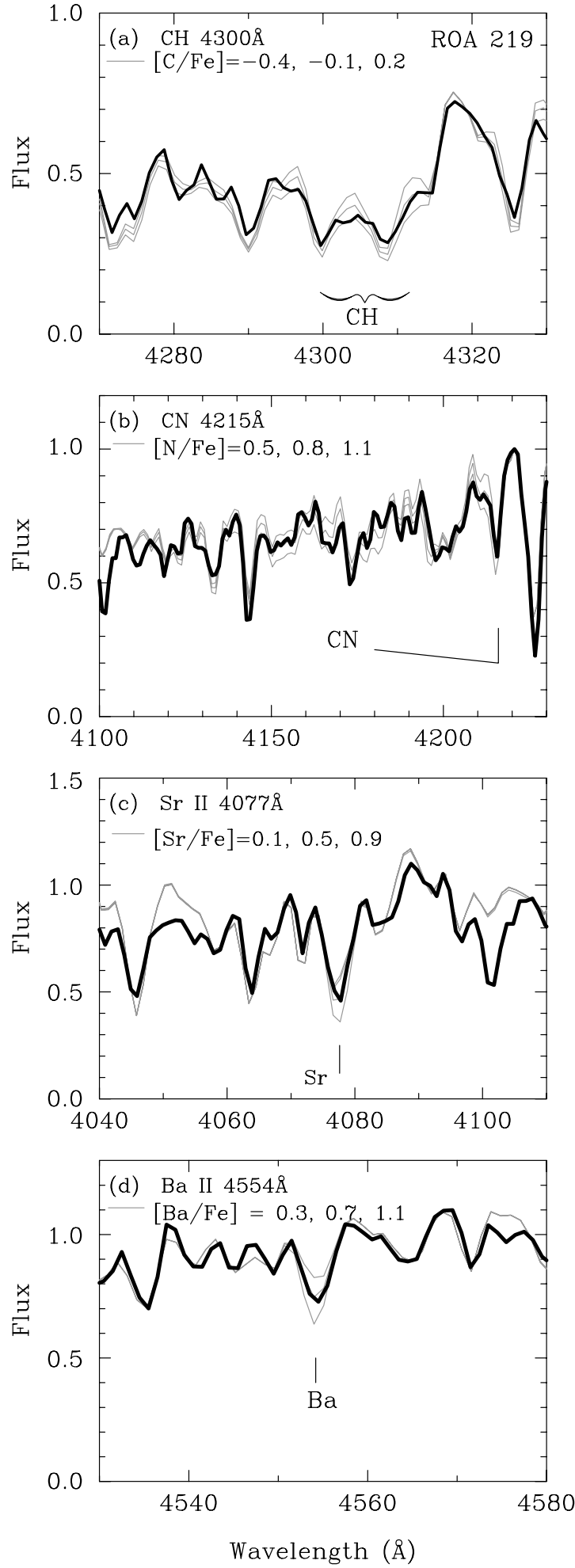

Figure 1. Observed spectrum (black line) of ROA 219 with synthetic spectra (gray lines). It has a resolution of $2.2 \AA$. The synthetic spectra in panel (a) have $[\mathrm{C} / \mathrm{Fe}]=-0.4,0.0,0.2$. The synthetic spectra in panel (b) adopt $[\mathrm{N} / \mathrm{Fe}]=0.5,0.8,1.1$, with $[\mathrm{C} / \mathrm{Fe}]=-0.1$. Panels (c) and (d) show synthetic spectra with $[\mathrm{Sr} / \mathrm{Fe}]=0.1,0.5,0.9$, and $[\mathrm{Ba} / \mathrm{Fe}]=0.3,0.7,1.1$, respectively.

The RGB star ROA 279 is a known CH star (ND95). No previous carbon or nitrogen abundance determinations could be found in the literature for this object (ND95 analyzed lines of other elements but did not report carbon, nitrogen or oxygen
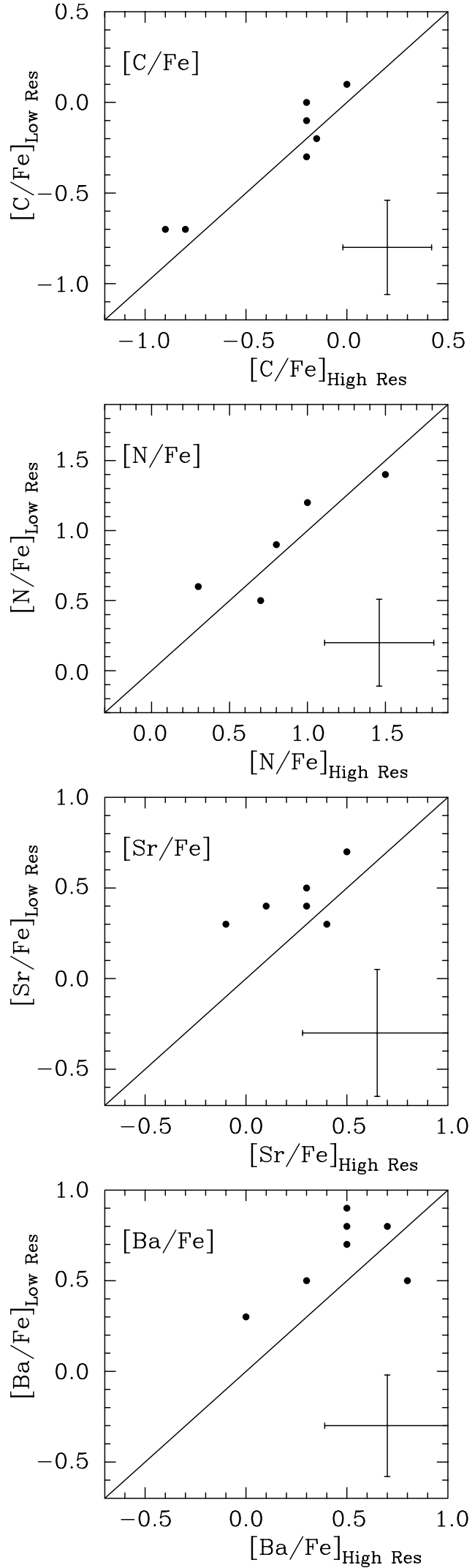

Figure 2. Comparison between R600 and R1200 carbon, nitrogen, strontium, and barium abundances for stars in common.

abundances). The $\mathrm{O}$ abundance for ROA 279 is not known and as this has an impact on the derived $\mathrm{C}$ abundance, an analysis was performed to determine its effect. Using oxygen 

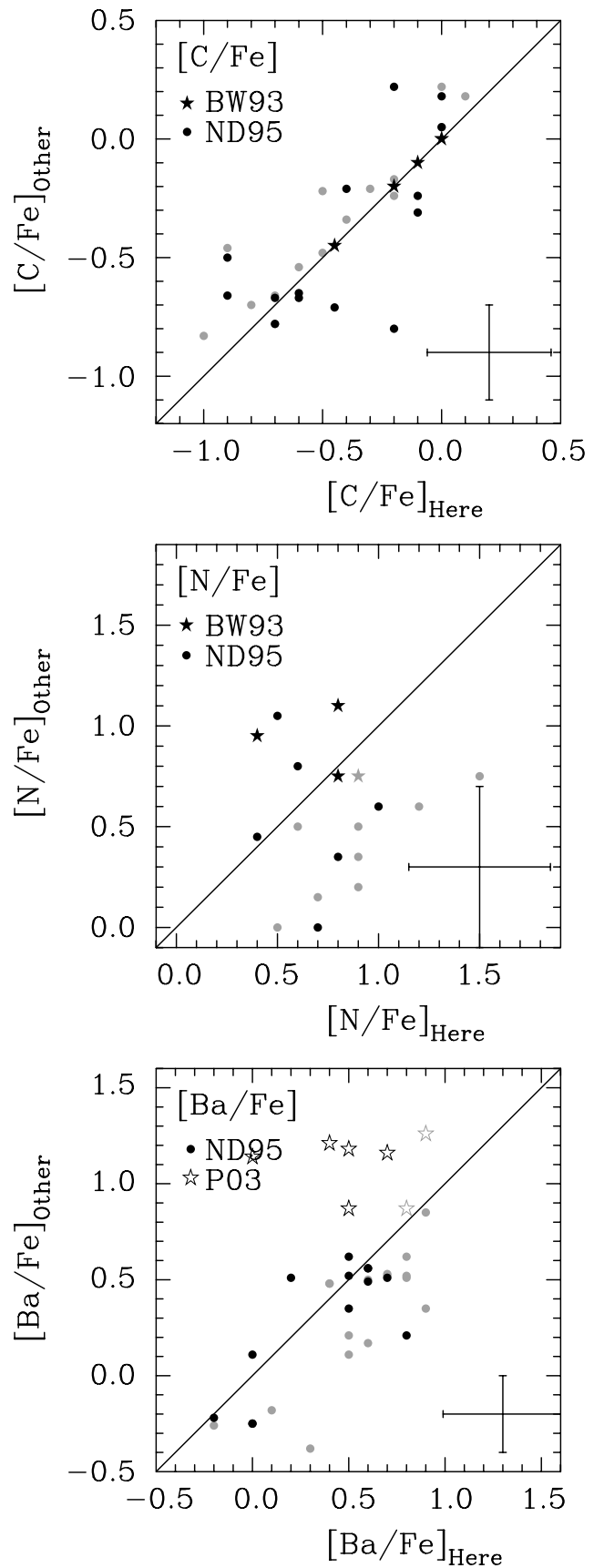

Figure 3. Carbon, nitrogen, and barium abundance comparisons between the R600 work (gray) and R1200 (black) on one hand, and ND95, BW93, and PAN03 on the other. The filled dots represent the comparisons between this work and ND95, the filled stars for BW93, and the open stars for PAN03. $\Delta[\mathrm{X} / \mathrm{Fe}]=[\mathrm{X} / \mathrm{Fe}]_{\text {This study }}-[\mathrm{X} / \mathrm{Fe}]_{\text {Other studies }}$.

abundances in the range $[\mathrm{O} / \mathrm{Fe}]=-0.6$ to +0.6 led to a spread in $\mathrm{C}$ of $0.8 \mathrm{dex}$, with $[\mathrm{C} / \mathrm{Fe}]=-0.1$ to +0.7 . The oxygen abundance was assumed to be $[\mathrm{O} / \mathrm{Fe}]=0.3$, and this led to $[\mathrm{C} / \mathrm{Fe}]=0.6$ and $[\mathrm{N} / \mathrm{Fe}]=0.5$. The $\mathrm{Sr}$ and $\mathrm{Ba}$ abundances determined for ROA 279 were $[\mathrm{Sr} / \mathrm{Fe}]=[\mathrm{Ba} / \mathrm{Fe}]=0.6$. Bell \& Dickens (1974) analyzed two CH stars, ROA 55 and ROA 70, in $\omega$ Cen, and found $[\mathrm{C} / \mathrm{H}]=-0.8,[\mathrm{~N} / \mathrm{H}]=0.0$ and assumed $[\mathrm{O} / \mathrm{H}]=-0.3$. Adopting $[\mathrm{Fe} / \mathrm{H}]=-1.9$ for ROA 70 (Gratton 1982), the carbon and nitrogen abundance ratios are $[\mathrm{C} / \mathrm{Fe}]=1.0$ and $[\mathrm{N} / \mathrm{Fe}]=1.8$. While the abundance of $\mathrm{C}$ found here $([\mathrm{C} / \mathrm{Fe}]=0.6)$ for ROA 279 is less than these $\mathrm{CH}$ stars analyzed by Bell \& Dickens (1974), it is still considerably
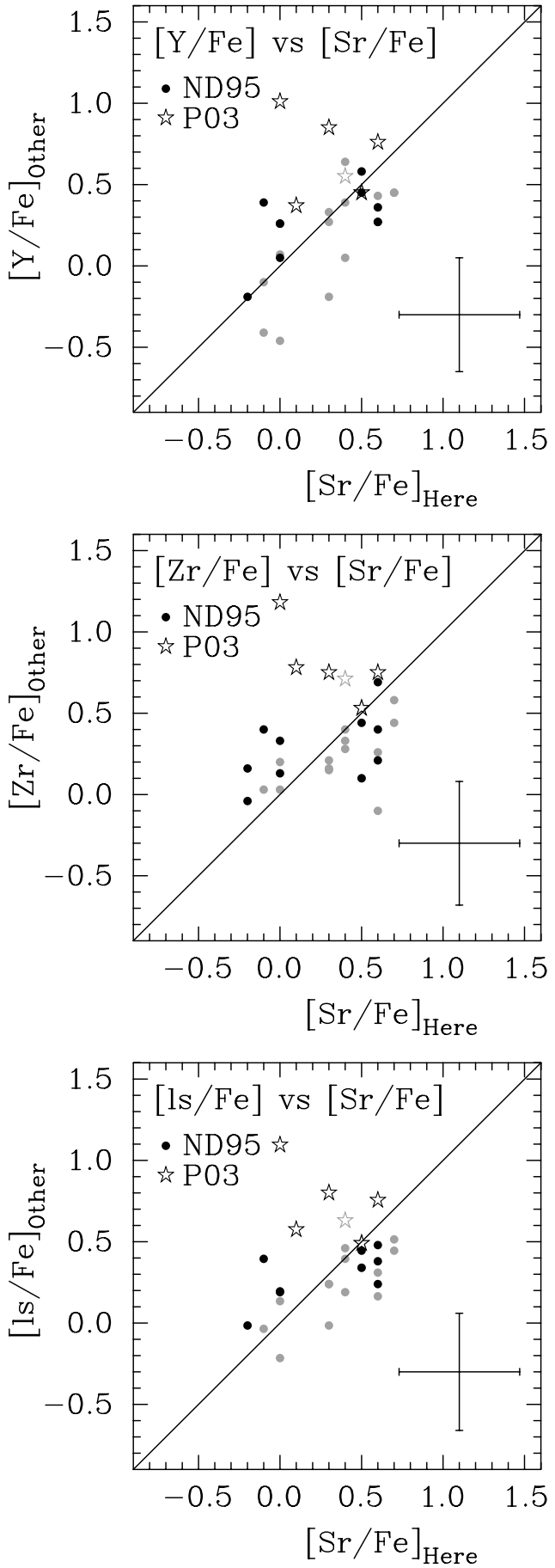

Figure 4. Light s-process element (Y, Zr) comparison between the R600 work (gray dots) and R1200 work (black dots) on one hand, and ND95 (filled dots) and PAN03 (open stars) on the other. [ls/Fe] is an average of abundances of $[\mathrm{Zr} / \mathrm{Fe}]$ and $[\mathrm{Y} / \mathrm{Fe}] . \Delta[\mathrm{X} / \mathrm{Fe}]=[\mathrm{X} / \mathrm{Fe}]_{\text {This study }}-[\mathrm{X} / \mathrm{Fe}]_{\text {Other studies. }}$. Please note that the filled star in the top panel is actually an unfilled star and a filled dot at the same point.

higher than in other RGB stars in $\omega$ Cen, including the COstrong objects. The enhancement of the $s$-process elements is comparable to that of the other $\mathrm{CH}$ stars, ROA 55 and 70 (Gratton 1982), field CH stars and other stars with s-process enhancement in the cluster. The nitrogen abundances obtained, however, are over 1 dex different. This is an interesting discrepancy and warrants further investigation. The enrichment of $\mathrm{C}$ and $s$-process material in field $\mathrm{CH}$ stars is generally thought to come 

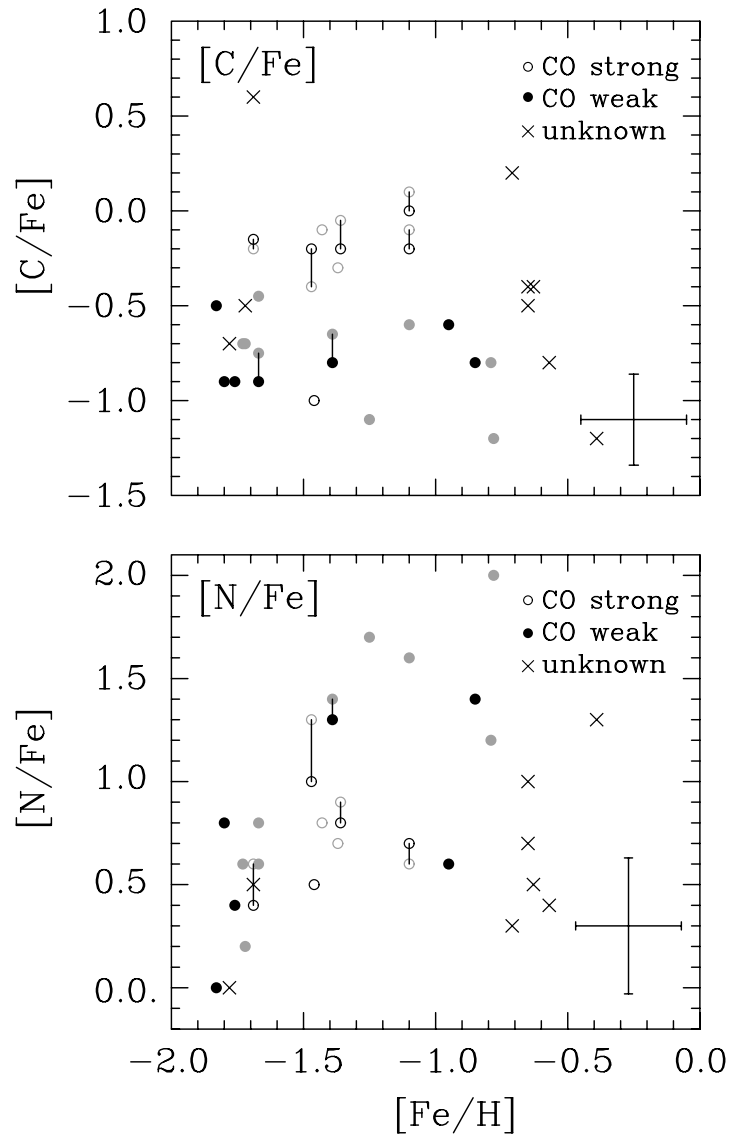

Figure 5. Carbon and nitrogen abundances plotted as functions of metallicity for the R600 (gray dots) and R1200 (black dots) resolution data. The open circles represent the $\mathrm{CO}$-strong stars, and closed circles represent the $\mathrm{CO}$-weak stars. The stars for which there is no information regarding the $\mathrm{CO}$ nature are represented by crosses. Abundances of stars in common between the R600 and R1200 resolution analysis are joined by solid lines.

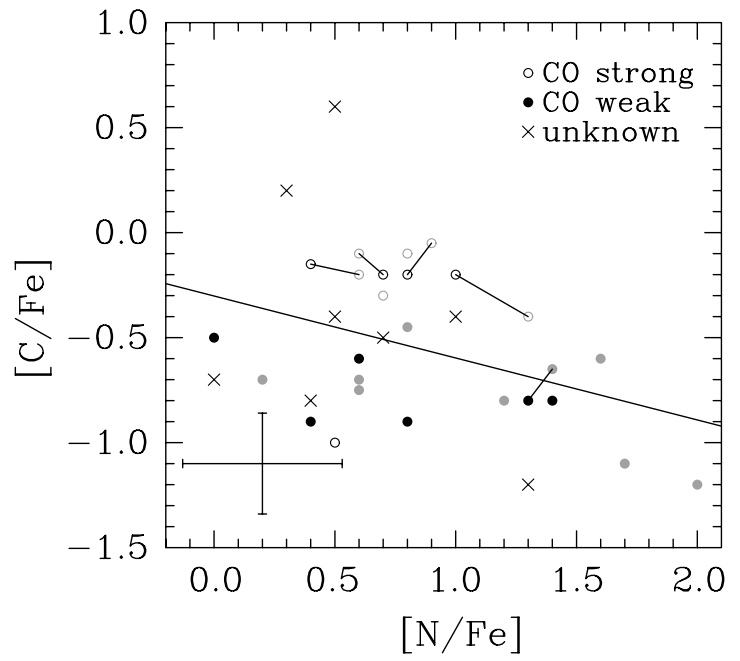

Figure 6. Carbon abundance plotted as a function of nitrogen abundance for the R600 (gray) and (black) resolution samples. The open circles represent the CO-strong stars, and closed circles represent the CO-weak stars. The stars for which there is no information regarding the $\mathrm{CO}$ nature are represented by crosses. Abundances of stars in common between the R600 and R1200 resolution analysis are joined by solid lines. The solid line represents the least-squares fit to the data.

from mass transfer in binary systems (McClure \& Woodsworth 1990), and may be the case here as well.
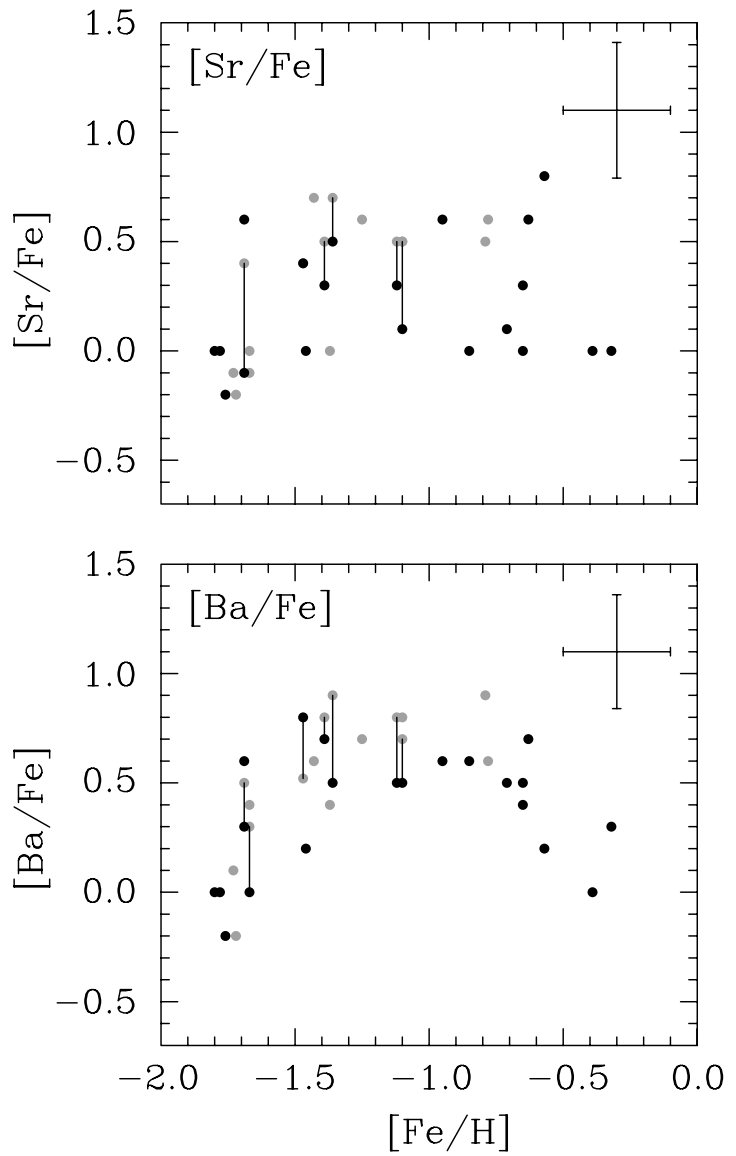

Figure 7. Strontium and barium abundances plotted as functions of metallicity for the R600 (gray) and R1200 (black) data. Abundances of stars in common between the R600 and R1200 resolution analysis are joined by solid lines.

\subsubsection{Strontium and Barium}

The $\mathrm{Sr}$ and $\mathrm{Ba}$ abundances are plotted as a function of metallicity in Figure 7 for both the R600 (gray) and R1200 (black) resolution data. The steep incline and then roughly constant value seen in the $\mathrm{Ba}$ abundance plot is similar to that seen in ND95. At higher metallicities, a downward trend in the $\mathrm{Ba}$ abundances is also seen which is not present in other studies. These objects are the most metal-rich ones in our sample, and several come from the studies of PAN03. In Section 4.3, a comparison was made between the barium abundances determined here and those from PAN03. It was found that for stars in common $[\mathrm{Ba} / \mathrm{Fe}]$ values determined here were lower than those found in PAN03 $\left(\left\langle[\mathrm{Ba} / \mathrm{Fe}]_{\mathrm{R} 600}\right\rangle \sim\right.$ -0.22 and $\left.\left\langle[\mathrm{Ba} / \mathrm{Fe}]_{\mathrm{R} 1200}\right\rangle \sim-0.69\right)$. This may account for the downward trend seen in the barium abundances at higher metallicities.

Figure 8 plots the strontium abundance against that of barium for the R600 (gray) and R1200 (black) resolution data. Objects in common between the two data sets are joined by a solid line. This plot shows a clear correlation between $[\mathrm{Sr} / \mathrm{Fe}]$ and $[\mathrm{Ba} / \mathrm{Fe}]$. A one-to-one line is drawn for reference as a dotted line. A least-squares fit was performed on the data, indicated by the solid line. The $p$-value for the least-squares fit was $<0.001$, indicating a very high probability that there is a correlation between the strontium and barium abundances. ${ }^{3}$

\footnotetext{
3 In this case, the null hypothesis was that the $[\mathrm{Sr} / \mathrm{Fe}]$ and $[\mathrm{Ba} / \mathrm{Fe}]$ abundances were unrelated.
} 


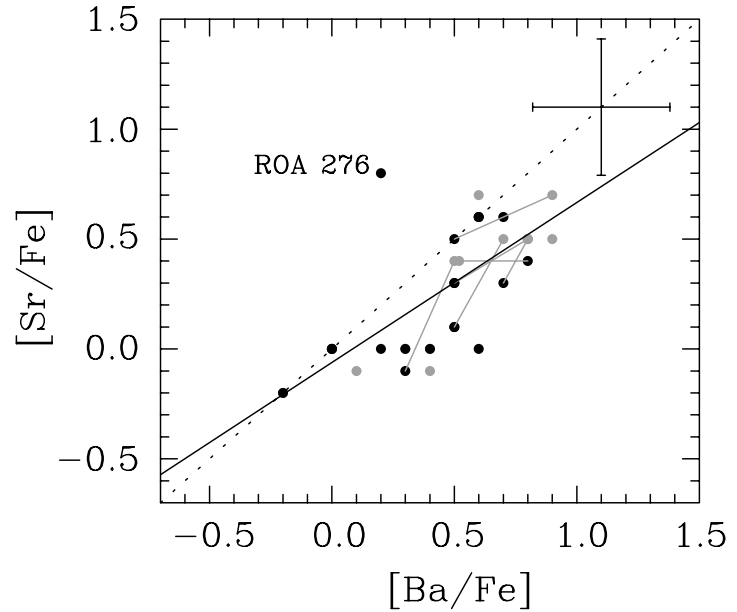

Figure 8. Strontium abundance plotted as a function of barium abundance for the R600 (gray) and R1200 (black) resolution samples. Abundances of stars in common between the R600 and R1200 analysis are joined by solid lines. The solid line is the least-squares fit to the data. The dotted line is the 1:1 line for reference.

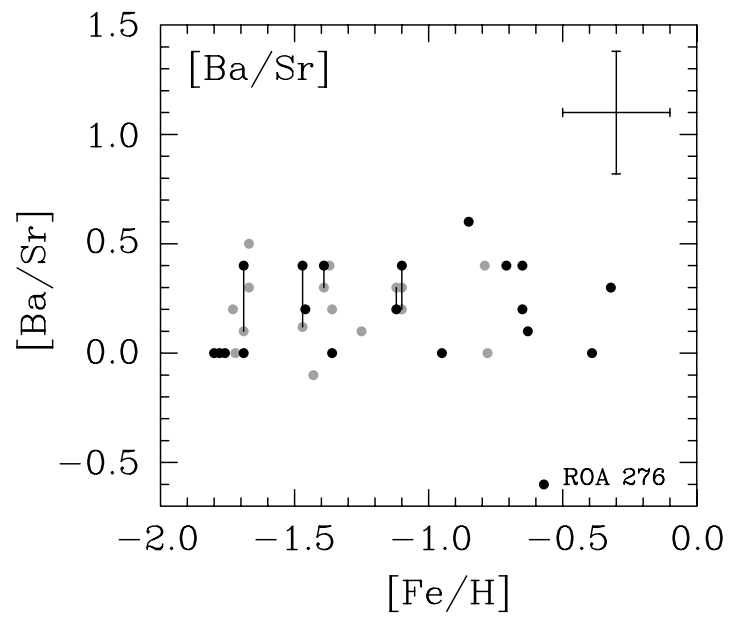

Figure 9. Ratio of heavy to light neutron-capture elements plotted as a function of metallicity for the R600 (gray) and R1200 (black) samples. Abundances of stars in common between the R600 and R1200 analysis are joined by solid lines.

The ratio of the heavy s-process elements (e.g., Ba) to light ones (e.g., Sr) gives an indication of the level of neutron exposure, with a higher value indicating a higher exposure. Figure 9 plots the ratio $[\mathrm{Ba} / \mathrm{Sr}]$, designated here as $[\mathrm{hs} / \mathrm{ls}]$, as a function of $[\mathrm{Fe} / \mathrm{H}]$. Most objects have $[\mathrm{hs} / \mathrm{ls}] \geqslant 0.0$, with one exception, ROA 276, described in more detail in the following section. This star shows the surprisingly low value $[\mathrm{Ba} / \mathrm{Sr}]=[\mathrm{hs} / \mathrm{ls}]=-0.6$.

\subsubsection{ROA 276}

While most stars in the sample had measured $[\mathrm{Ba} / \mathrm{Fe}]$ equal to or greater than $[\mathrm{Sr} / \mathrm{Fe}], \mathrm{ROA} 276$ deviates from this general trend with significantly larger $[\mathrm{Sr} / \mathrm{Fe}]$ than $[\mathrm{Ba} / \mathrm{Fe}]$, as seen in Figure 8 . The reader may recall that a similar $[\mathrm{Ba} / \mathrm{Sr}]$ ratio was found before in the $\omega$ Cen MS star S2015448, described in Stanford et al. (2006b). ROA 276 is metal-rich with $[\mathrm{Fe} / \mathrm{H}]=-0.57$, has depleted carbon $([\mathrm{C} / \mathrm{Fe}]=-0.80)$, and enhanced nitrogen $([\mathrm{N} / \mathrm{Fe}]=0.4)$. The observed and synthetic spectra in the region of Sr II $4077 \AA$ and Ba II $4554 \AA$ are shown in Figure 10.

The stellar parameters for this object were derived by interpolation of $T_{\text {eff }}$ and $\log g$ given its metallicity converted from
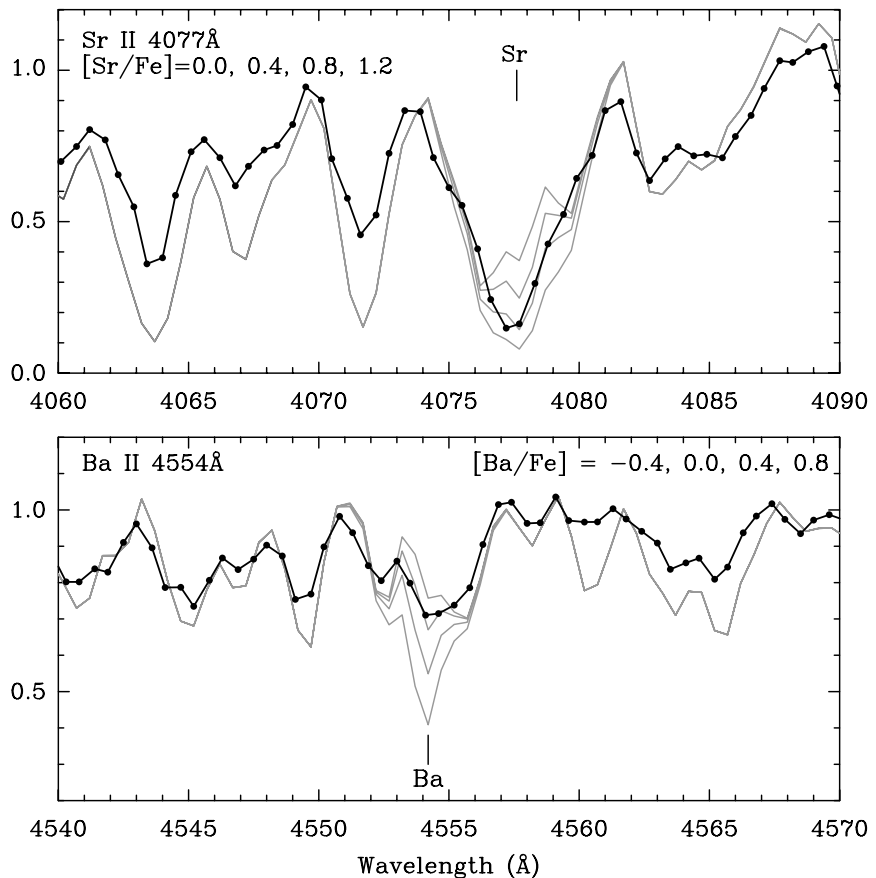

Figure 10. Observed 1200 I grating spectrum of ROA 276 (black line) with synthetic spectra (gray lines). The stellar parameters used were $T_{\text {eff }}=4000$, $\log g=0.7,[\mathrm{Fe} / \mathrm{H}]=-0.57$, and $v_{t}=2.0$.

$[\mathrm{Ca} / \mathrm{H}]$ (NFM96) as described in Section 3. When analyzing the Sr II $4077 \AA$ feature in the observed spectra it was noticed that many of the neighboring lines were too strong, indicating the metallicity was too high or the temperature too low. By decreasing the metallicity to $[\mathrm{Fe} / \mathrm{H}]=-1.4$, and redetermining the temperature to be $4200 \mathrm{~K}$ (as opposed to $4000 \mathrm{~K}$ ), an improved fit to these lines was found. The effect this had on the abundances was to increase $[\mathrm{Sr} / \mathrm{Fe}]$ from 0.8 to 1.8 and $[\mathrm{Ba} / \mathrm{Fe}]$ from 0.2 to 0.6. Despite the change in stellar parameters the unusual $[\mathrm{Ba} / \mathrm{Sr}]$ ratio is still present. Although the original stellar parameters and abundances have been recorded in each of Tables 3 and 4, it should be kept in mind that the temperature and metallicity have been estimated and require more accurate determination. Further analysis of this object is required to accurately determine its stellar parameters and to confirm the $s$-process abundance pattern found here.

As a consequence of the abundance result obtained with the intermediate-resolution spectra, higher resolution data were obtained for ROA 276 along with two other $\omega$ Cen RGB stars, ROA 46 and ROA 150. These data were obtained with the Magellan (Clay) telescope/MIKE echelle spectrograph combination (see http://www.ucolick.org/ rab/MIKE/usersguide.html) during 2007 June $22-23$. We used a 0'.5 slit and resolving power of 47,000 . The resolution of the data is $0.12 \AA$ FWHM. Here we concentrate on the wavelength regions $4065-4085 \AA$ and $4545-4565 \AA$ to cover the Sr II $4077 \AA$ line and the Ba II $4554 \AA$ one. Hyperfine splitting for the Ba II $4554 \AA$ line was taken into account and followed the $s$-process abundance pattern from Arlandini et al. (1999).

The synthetic spectra were produced using the models and line lists employed for the $2.3 \mathrm{~m}$ spectra as described in Section 4. As mentioned previously, the stellar parameters are not well known for ROA 276. Synthetic spectra produced using the parameters determined above $\left(T_{\text {eff }}=4000 \mathrm{~K}, \log g=0.7\right.$, $[\mathrm{Fe} / \mathrm{H}]=-0.57)$ did not match the observed spectra very well with the lines produced in the synthetic spectra being 

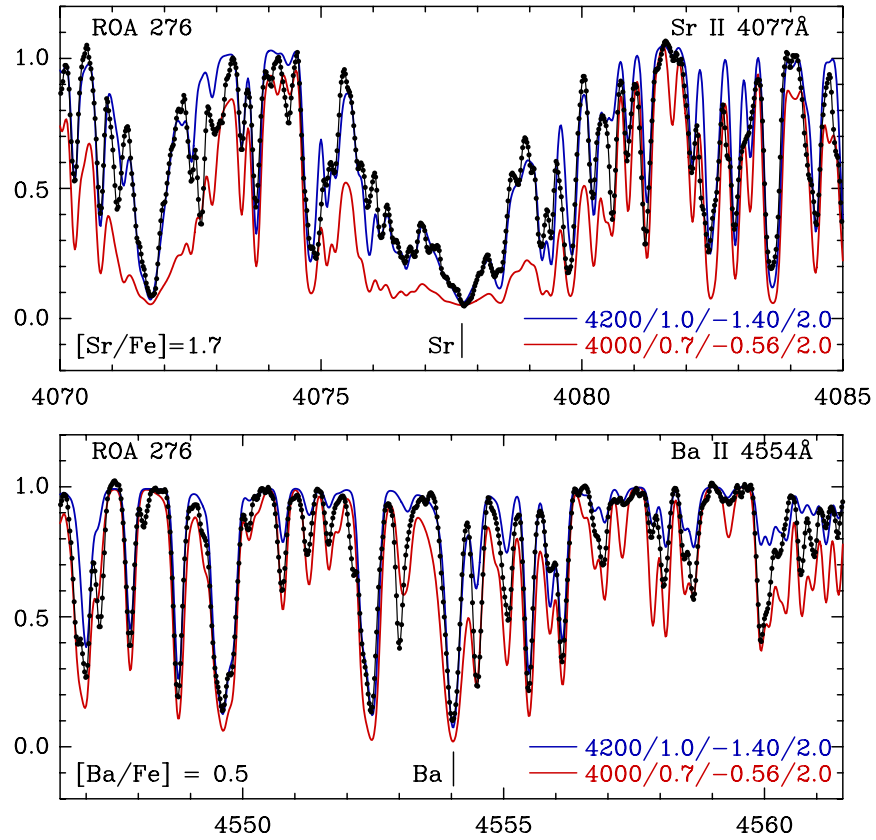

Figure 11. High-resolution observed MIKE spectrum of ROA 276 (black dotted line) plotted with two synthetic spectra with differing stellar parameters. The solid blue line has $T_{\text {eff }} / \log g /[\mathrm{Fe} / \mathrm{H}] / v_{t}=4200 / 1.0 /-1.40 / 2.0$ and the red line has $T_{\text {eff }} / \log g /[\mathrm{Fe} / \mathrm{H}] / v_{t}=4000 / 0.7 / 0.56 / 2.0$.

(A color version of this figure is available in the online journal.)

Table 5

$[\mathrm{Ba} / \mathrm{Sr}]$ Abundances Obtained from Magellan/MIKE Data as a Function of Temperature and Metallicity for ROA 276

\begin{tabular}{lcccc}
\hline \hline$[\mathrm{Fe} / \mathrm{H}]$ & \multicolumn{3}{c}{$T_{\text {eff }}$} \\
\cline { 2 - 5 } & 3800 & 4000 & 4200 & 4400 \\
\hline-0.6 & -1.40 & -1.10 & -1.45 & -1.40 \\
-1.0 & -1.20 & -1.15 & -1.40 & -1.30 \\
-1.4 & -1.10 & -1.30 & -1.20 & -1.35 \\
\hline
\end{tabular}

deeper than those observed. To illustrate this point, in Figure 11 we compare the observed MIKE high-resolution data for both Sr II $4077 \AA$ (upper panel) and Ba II 4554 A (lower panel) with the synthetic spectra having stellar parameters $\left(T_{\text {eff }}=4000 \mathrm{~K}\right.$, $\log g=0.7,[\mathrm{Fe} / \mathrm{H}]=-0.57)$ and $\left(T_{\text {eff }}=4200 \mathrm{~K}, \log g=1.0\right.$, $[\mathrm{Fe} / \mathrm{H}]=-1.4)$ in both panels.

To test the robustness of the results based on the stellar parameters, synthetic spectra were produced with a range in temperature and metallicity (surface gravity corresponded to the temperature based on the star's position in the CMD). Four effective temperatures $\left(T_{\text {eff }}=3800 \mathrm{~K}, 4000 \mathrm{~K}, 4200 \mathrm{~K}, 4400 \mathrm{~K}\right)$ and three metallicities $([\mathrm{Fe} / \mathrm{H}]=-0.57,-1.0,-1.4)$ were used. The $[\mathrm{Ba} / \mathrm{Sr}]$ ratio ranges between -1.1 and -1.4 for each of these models. As shown in Table $5,[\mathrm{Ba} / \mathrm{Sr}]$ is largely independent of the input stellar parameters. More accurate stellar parameters will be determined in a forthcoming paper where the red high-resolution MIKE data are analyzed and results are presented.

Figure 12 shows the $\mathrm{Sr} 4077 \AA$ A line for ROA 40 (panel a), 150 (panel b), and 276 (panel c). The results for the Ba $4554 \AA$ line are shown for all three stars in Figure 13. (For ROA 150, one will notice a flat-bottom in the line profile for the Ba $4554 \AA$ line. The exact cause of this is unclear, but it is suspected to be due to saturation effects.) For comparison, in both figures a synthetic spectrum with stellar parameters $T_{\text {eff }}=4200 \mathrm{~K}$,
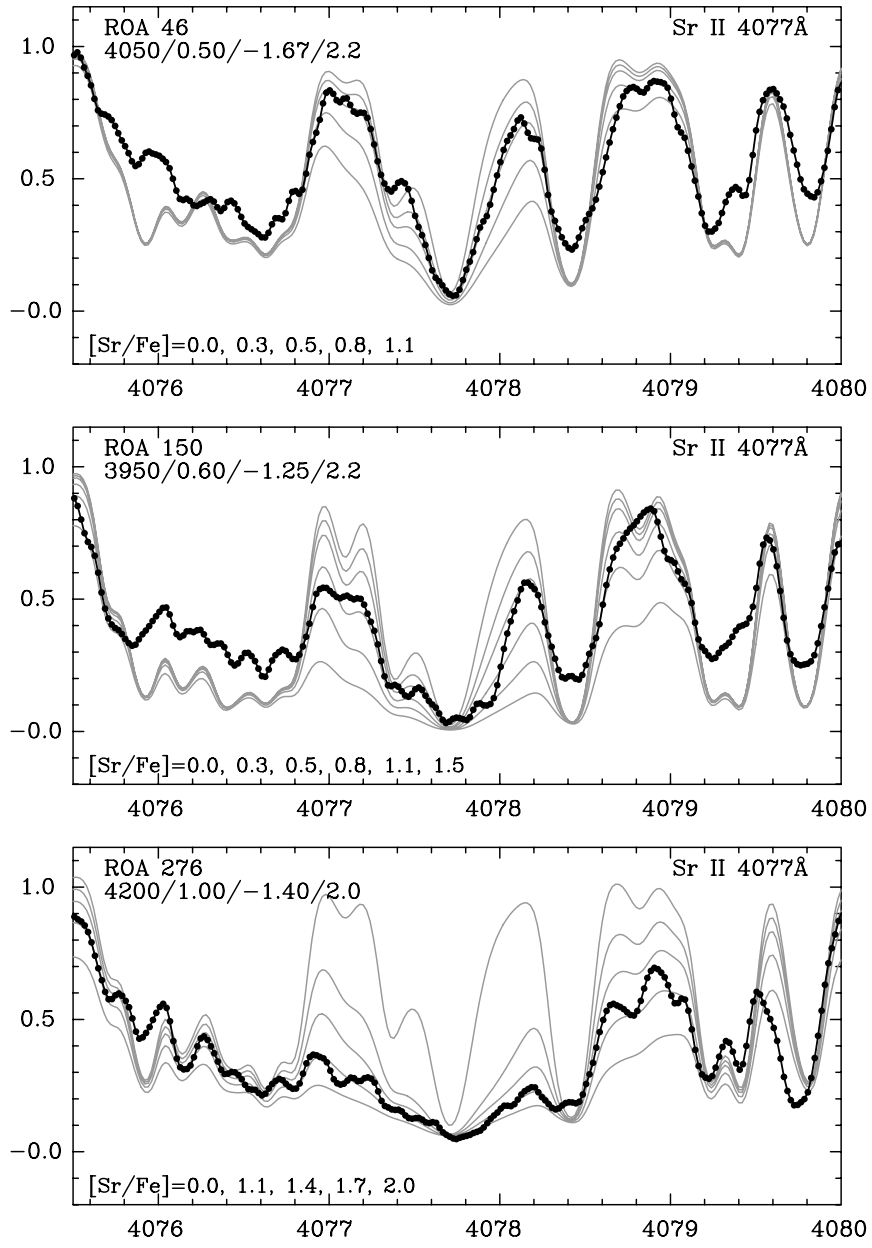

Figure 12. High-resolution observed MIKE spectra of ROA 46, 150, and 276 (black, dotted line) showing the synthetic spectra fit (gray lines) to the $\mathrm{Sr} 4077 \AA$ line. The synthetic spectra have stellar parameters: $T_{\text {eff }}=4050, \log g=0.50$, $[\mathrm{Fe} / \mathrm{H}]=-1.67$, and $v_{t}=2.2$ for ROA 46; $T_{\text {eff }}=3950, \log g=0.60$, $[\mathrm{Fe} / \mathrm{H}]=-1.25$, and $v_{t}=2.2$ for ROA 150 ; and $T_{\text {eff }}=4200, \log g=1.00$, $[\mathrm{Fe} / \mathrm{H}]=-1.40$, and $v_{t}=2.0$ for ROA 276 .

Table 6

[Sr/Fe] and $[\mathrm{Ba} / \mathrm{Fe}]$ Abundances Using MIKE High-resolution Spectra for Three $\omega$ Cen RGB Stars-ROA 46, 150, and 276

\begin{tabular}{lccr}
\hline \multicolumn{1}{c}{ Star } & {$[\mathrm{Sr} / \mathrm{Fe}]$} & {$[\mathrm{Ba} / \mathrm{Fe}]$} & {$[\mathrm{Ba} / \mathrm{Sr}]$} \\
\hline ROA 46 & 0.4 & 0.15 & -0.25 \\
ROA 150 & 0.6 & 0.70 & 0.10 \\
ROA 276 & 1.6 & 0.40 & -1.20 \\
\hline
\end{tabular}

$\log g=1.0,[\mathrm{Fe} / \mathrm{H}]=-1.40$, and $v_{t}=2.0 \mathrm{~km} \mathrm{~s}^{-1}$ have been plotted for a range of $[\mathrm{Sr} / \mathrm{Fe}]$ and $[\mathrm{Ba} / \mathrm{Fe}]$ values against the observed ROA 276 one. These were chosen as they gave the best fit to the observed spectra. Table 6 lists the $[\mathrm{Sr} / \mathrm{Fe}]$ and $[\mathrm{Ba} / \mathrm{Fe}]$ results for each of the three stars as well as the $[\mathrm{Ba} / \mathrm{Sr}]$ abundance. It is clear from the MIKE high-resolution data that ROA 276 has an unusual $[\mathrm{Ba} / \mathrm{Sr}]$ ratio, and that further study of this star is warranted.

\section{DISCUSSION}

The abundances found for the RGB stars here cannot only provide information about enrichment sources for these stars, but also by comparing the abundances with those found for the MSTO, further constraints can be derived. Comparing the MSTO and RGB abundance patterns may also show how these 

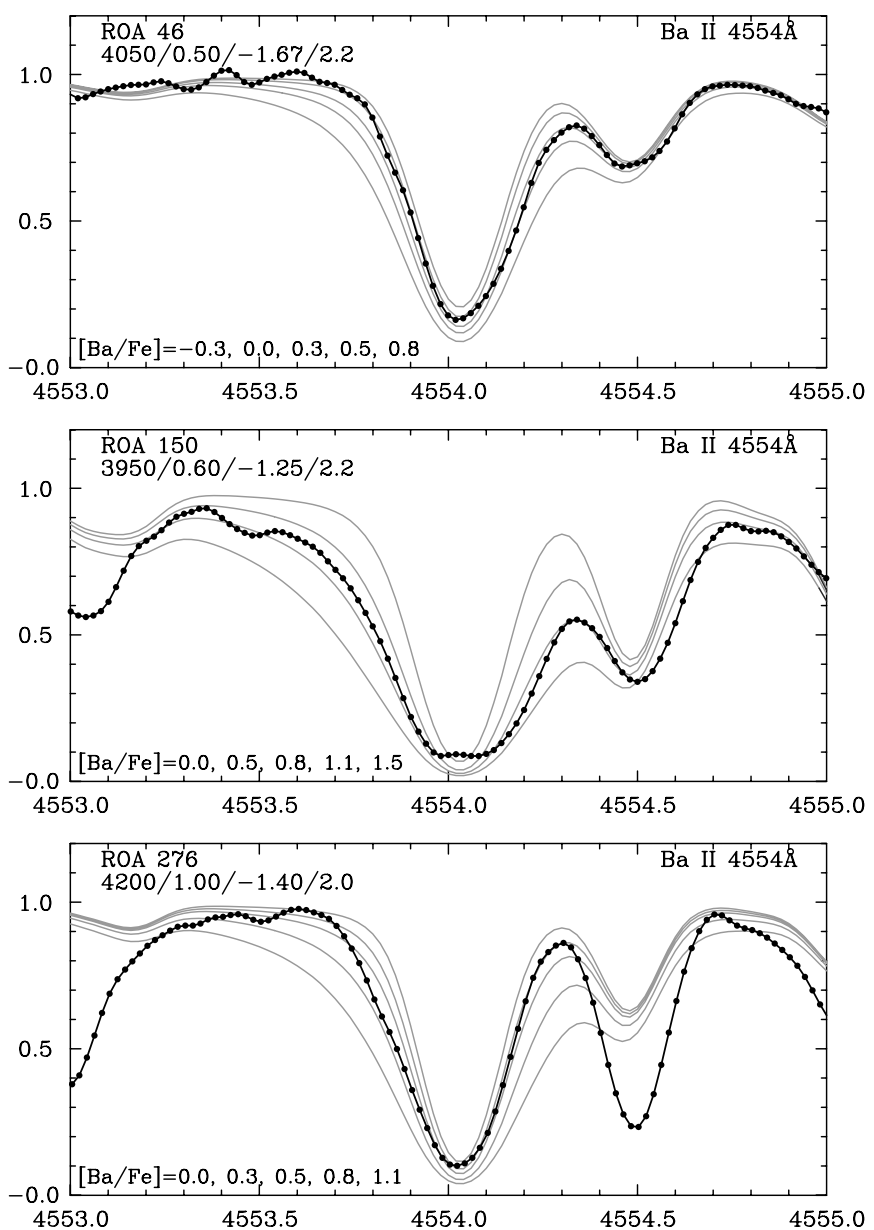

Figure 13. High-resolution MIKE spectra of ROA 46, 150, and 276 (black, dotted line) showing the synthetic spectra fit (gray lines) to the Ba $4554 \AA$ line. The synthetic spectra have stellar parameters: $T_{\text {eff }}=4050, \log g=0.50$, $[\mathrm{Fe} / \mathrm{H}]=-1.67$, and $v_{t}=2.2$ for ROA 46; $T_{\text {eff }}=3950, \log g=0.60$, $[\mathrm{Fe} / \mathrm{H}]=-1.25$, and $v_{t}=2.2$ for ROA 150 ; and $T_{\mathrm{eff}}=4200, \log g=1.00$, $[\mathrm{Fe} / \mathrm{H}]=-1.40$, and $v_{t}=2.0$ for ROA 276 .

Table 7

Comparisons of Abundances Obtained from R600 2.3 m Data for ROA 276 with those of S2015448

\begin{tabular}{cccccc}
\hline \hline ID & {$[\mathrm{Fe} / \mathrm{H}]$} & {$[\mathrm{C} / \mathrm{Fe}]$} & {$[\mathrm{N} / \mathrm{Fe}]$} & {$[\mathrm{Sr} / \mathrm{Fe}]$} & {$[\mathrm{Ba} / \mathrm{Fe}]$} \\
\hline ROA 276 & -0.57 & -0.80 & 0.4 & 0.8 & 0.2 \\
S2015448 & -0.74 & -0.50 & $<0.5$ & 1.6 & $<0.6$ \\
\hline
\end{tabular}

enrichments originated-that is, whether they are primordial, evolutionary or the material was accreted onto the surface layers of the stars.

As a star ascends the RGB, the convective envelope deepens considerably and material that was processed via the $\mathrm{CN}$ cycle in the interior of the star is possibly mixed to the surface layers (see Sweigart \& Mengel 1979). This would result in less carbon and more nitrogen when compared with earlier evolutionary stages, with the overall $\mathrm{C}+\mathrm{N}$ abundance remaining constant. In this instance, the abundances of the $s$-process elements are not changed. The level of mixing, if any, on the RGB is, however, uncertain. In some globular clusters there is evidence of mixing as stars ascend the RGB as the carbon and nitrogen abundances are different from the MS level compared with those at the tip of the RGB (Langer et al. 1986; Briley et al. 2002). An example of such a cluster is M13 (Smith \& Briley 2006 and references therein). It shows chemical inhomogeneities for elements $\mathrm{C}$ through $\mathrm{Al}$, and is a result of a primordial abundance spread, coupled with mixing on ascent of the RGB. On the other hand, other globular clusters, such as 47 Tuc show no difference in the abundance patterns of carbon and nitrogen between the MS and RGB (Cannon et al. 1998; Briley et al. 2004; Da Costa et al. 2004).

A competing factor to the mixing process is the enhancements of carbon, nitrogen, and $s$-process elements in stars on the MSTO of $\omega$ Cen. These enhancements arose either from primordial enrichment (the enhanced material is uniform throughout the star's interior) or accretion events (the enhanced material is in the surface layers only). In the case of the enhanced material being accreted, as a star ascends the RGB, the convective envelope deepens and the enhanced material is mixed with unenhanced material. This results in a dilution of the enhanced material which may include carbon, nitrogen, and $s$-process elements. Alternatively, if the material is uniform throughout the star, the same abundances for the MSTO and RGB stars should be observed. That said, the evolutionary mixing, described above, adds a further complication to the picture and must be taken into consideration when attempting to decipher the differences between the MSTO and RGB.

Another type of mixing to take into consideration is thermohaline mixing (Kippenhahn et al. 1980; Charbonnel \& Zahn 2007). This process involves mixing of material that has been accreted when the mean molecular weight of the stellar gas increases toward the surface of the star. The gas is displaced downwards and compressed, making it hotter than its surroundings. This in turn makes it lose more heat, become denser, and continue to sink. This then leads to mixing on thermal timescales. Stancliff \& Glebbeek (2008) estimate thermohaline processes mixing the accreted material with $16 \%-88 \%$ of the pristine gas. This, however, was for a model of a star with $Z=10^{-4}([\mathrm{Fe} / \mathrm{H}]=-2.3)$, whereas most of the enhanced stars are more metal-rich than this by at least 1 dex. Therefore, the difference in mean molecular weight is smaller, and thermohaline mixing to be less efficient. Several other studies have found the thermohaline process to be less efficient that first thought. In a comparison between turnoff stars and red giants, Aoki et al. (2008) showed that the distribution of $[\mathrm{C} / \mathrm{Fe}]$ was different between the two sets, suggesting significant mixing only occurs at the first dredge up. Denissenkov \& Pinsonneault (2008) reach a similar conclusion using the data from Lucatello et al. (2006), where they found the $[\mathrm{C} / \mathrm{H}]$ reduced by $\sim 0.4$ dex resulting from the first dredge up.

It is interesting to estimate the dilution factor-the amount surface pollution is diluted as the convective envelope deepens on ascent of the RGB. This factor depends on the metallicity and mass of the star (Yoshii 1981) as these influence the mass fraction of the convective envelope on the MS and RGB. The convective envelope mass faction increases for increasing metallicity, and decreasing stellar mass.

The dilution factor can then be calculated, given by the following equation:

$$
[\mathrm{X} / \mathrm{Fe}]_{\mathrm{RGB}}=\log \left[1+\left(10^{[\mathrm{X} / \mathrm{Fe}]} \mathrm{MS}-1\right) \frac{m_{\mathrm{MS}}}{m_{\mathrm{RGB}}}\right],
$$

where $[\mathrm{X} / \mathrm{Fe}]_{\mathrm{MS}}$ is the abundance of element $\mathrm{X}$ in the surface layers before dilution, $[\mathrm{X} / \mathrm{Fe}]_{\mathrm{RGB}}$ is the abundance of element $\mathrm{X}$ on the RGB after dilution, and the mass fractions of the convective envelope on the MS and RGB are represented by $m_{\mathrm{MS}}$ and $m_{\mathrm{RGB}}$, respectively. The interior of the MS star is assumed to have a solar abundance ratio of $[\mathrm{X} / \mathrm{Fe}]$. Using the convective envelope mass fractions of $m_{\mathrm{MS}}=2.256 \times 10^{-2}$ 
and $m_{\mathrm{RGB}}=0.6837$ for an $0.8 M_{\odot}$ star with initial metallicity $[\mathrm{Fe} / \mathrm{H}]=-0.75$ and enhanced $\alpha$-elements (D. VandenBerg 2009 , private communication), the depletion factor is found to be $\sim 30$. Using envelope mass fractions for lower metallicity stars, larger mass stars, or lower $\alpha$-element enhancement all result in greater depletion values. The models of L. Girardi (2009, private communication) give similar results. This dilution factor indicates that any surface contamination would be diluted to a large degree, although still measurable on the RGB. The inclusion of thermohaline mixing would only serve to increase the abundance one would find on the MS using the RGB abundance ratios.

There are several possible enrichment sources for the enhancements. One possibility is low-mass $\left(\lesssim 3 M_{\odot}\right)$ AGB stars, the products of which are carbon and $s$-process elements (Gallino et al. 1998). Intermediate-mass $\left(3 \lesssim M_{\odot} \lesssim 8\right)$ AGB stars may also play a role, producing nitrogen and perhaps a small amount of $s$-process elements (depending on the size of the ${ }^{13} \mathrm{C}$ pocket; Ventura et al. 2002). Massive stars may also contribute to the enrichment, in particular massive rotating objects. These produce excess helium and nitrogen in their stellar winds without carbon and oxygen overabundances (Maeder \& Meynet 2006). These stars may be able to account for the abundances found on the RGB.

Those objects with near solar abundance ratios of carbon and supersolar s-process element enrichment have possibly been enriched by low-mass AGB stars. Yet objects with enrichment in nitrogen are more consistent with enhancement from intermediate-mass AGB stars. As many of the nitrogen enhanced objects also show enrichment in $s$-process elements several different sources may be required to explain the observed abundance patterns. In these cases, rotating, massive stars may account for the $\mathrm{N}$ abundance patterns, while low-mass AGB stars could be the source of the $s$-process enhancements. As yet $s$-process element abundance yields for rotating, massive stars have not been published and therefore definitive conclusions regarding the enhancement sources of enriched nitrogen and strontium RGB stars cannot be made.

\subsection{Comparison of Abundances on the MSTO and RGB}

The RGB stars studied here do not have carbon abundances greater than $[\mathrm{C} / \mathrm{Fe}]=0.2$, except for the $\mathrm{CH}$ star $\mathrm{ROA}$ $279([\mathrm{C} / \mathrm{Fe}]=0.6)$, while few MSTO stars show depletions and several show enhancements $\left([\mathrm{C} / \mathrm{Fe}]_{\mathrm{MSTO}}=0.5-1.0\right)$. Consequently, the low $[\mathrm{C} / \mathrm{Fe}]$ values on the RGB may be due to the evolutionary effects during ascent of the RGB involving processing of $\mathrm{C}$ into $\mathrm{N}$. It should be kept in mind, however, that our ability to measure the $\mathrm{C}$ abundance for $[\mathrm{C} / \mathrm{Fe}]<0.5$ for the metal-poor and intermediate metallicity MSTO stars is not high; therefore, detailed comparisons, for example as a function of $[\mathrm{Fe} / \mathrm{H}]$, are not easily made.

Apart from the $\mathrm{CH}$ star ROA 279, many RGB stars are carbon depleted as would be expected from convective mixing during ascent of the RGB. However, inspection of Figure 6 shows a significant number of stars with $[\mathrm{C} / \mathrm{Fe}] \sim 0.0$, which were previously found in ND95 (the CO-strong objects of Persson et al. 1980), and in comparison with other globular clusters these objects are unique. ND95 provided three possible explanations for the carbon abundances of these stars: (1) they are only found in $\omega$ Cen due to the larger number of stars sampled in this cluster, (2) carbon depletion as a star ascends the RGB does not occur for all stars, and (3) depletion does occur in all stars, and the initial carbon abundance of those objects with near solar
$[\mathrm{C} / \mathrm{Fe}]$ was considerably higher than for the bulk of the population before its ascent of the RGB.

The carbon results found on the MSTO are consistent with the third possibility. The MSTO objects were found to have enhancements of $[\mathrm{C} / \mathrm{Fe}] \geqslant 0.5$ for $\sim 10 \%$ of stars (see Table 3, Stanford et al. 2007). ND95 suggest, in their consideration of the unbiased samples of Persson et al. (1980) and Cannon \& Stobie (1973), that within 1 mag of the tip of the RGB in $\omega$ Cen $5 \%-15 \%$ of the stars are CO-strong. As we do not find stars with carbon enhancements as great as those found at the MSTO on the upper RGB (except for the four $\mathrm{CH}$ stars), mixing to some degree must have occurred during the ascent of the RGB and resulted in objects with carbon abundances higher than found in the bulk of the main RGB population (i.e., $[\mathrm{C} / \mathrm{Fe}]>\sim-0.2$ ). ND95 reported depletion of carbon at the RGB tip in the range $\Delta[\mathrm{C} / \mathrm{Fe}]=0.3-1.0 \mathrm{dex}$, supporting the above result. Therefore, the carbon-rich objects on the MSTO could be the evolutionary precursors to the CO-strong stars on the RGB.

On inspection of Figure 6, it can be seen that those stars with the greatest depletions of carbon have the greatest enhancements of nitrogen. Those with near solar carbon abundance ratios have more modest nitrogen enhancements. This may be accounted for if the amount of evolutionary mixing a star goes though is not the same for every star for a given mass and metallicity. MS objects, on ascent of the RGB, may have processed little $\mathrm{C}$ into $\mathrm{N}$ resulting in RGB stars with near solar $[\mathrm{C} / \mathrm{Fe}]$, and moderate $\mathrm{N}$ enhancements. Alternatively, they may have processed a more substantial amount of carbon resulting in objects with low carbon abundances $([\mathrm{C} / \mathrm{Fe}] \sim-0.8)$, and large nitrogen enhancements. Nitrogen enhancements on the RGB show high $[\mathrm{N} / \mathrm{Fe}]=0.5-1.5$. This may be due to two sources. The first is primordial enrichment, the second $\mathrm{CN}$ cycling and mixing as the star ascends the RGB.

\subsubsection{Carbon-rich Stars}

Two MS C-enhanced stars, S7007334 ([Fe/H] = -1.84) and S9005309 $([\mathrm{Fe} / \mathrm{H}]=-1.88)$, were identified in Stanford et al. (2007) that may be the counterparts to the RGB CH stars in the cluster. They show enhancements in $[\mathrm{C} / \mathrm{Fe}]$ greater than those found for the $\mathrm{CH}$ star ROA $279([\mathrm{Fe} / \mathrm{H}]=-1.69)$ by 0.6 dex. These MS stars show no nitrogen or $s$-process enhancements that $\mathrm{CH}$ stars usually exhibit, although this may be due to the low sensitivity of our technique to these features at MS metallicities and temperatures.

On the subgiant branch (SGB), the star S8001811 (Stanford et al. 2007) is worthy of mention. Its carbon, nitrogen, and strontium abundance pattern $([\mathrm{C} / \mathrm{Fe}]=0.7,[\mathrm{~N} / \mathrm{Fe}]=1.2$, $[\mathrm{Sr} / \mathrm{Fe}]=1.4)$ is similar to that seen in the CH star ROA 279. This SGB object may be a precursor of the RGB CH stars in $\omega$ Cen, and a counterpart of the field "subgiant $\mathrm{CH}$ " stars of Luck \& Bond (1991).

\subsubsection{Nitrogen and s-process Enhanced Stars}

There exists a number of metal-rich stars $([\mathrm{Fe} / \mathrm{H}]>-1.1)$ on the MSTO that show enhancements in both nitrogen and $s$-process elements, together with low carbon, $([\mathrm{C} / \mathrm{Fe}] \sim-0.3$, $[\mathrm{N} / \mathrm{Fe}] \sim 1.8,[\mathrm{Sr} / \mathrm{Fe}] \sim 1.0$; Stanford et al. 2007). On the RGB, ROA 480 shows similar abundance patterns and is of similar metallicity with $[\mathrm{Fe} / \mathrm{H}]=-0.95$. With $[\mathrm{C} / \mathrm{Fe}]=-0.6$, its carbon abundance is depleted by a larger amount than that of the MSTO group. This difference may be due to the carbon having been processed into nitrogen as ROA 480 ascended the RGB. ROA 480 (with $[\mathrm{N} / \mathrm{Fe}]=0.6,[\mathrm{Sr} / \mathrm{Fe}]=0.6$ ) has lower 
nitrogen and strontium than found for the MSTO group. These abundance patterns with low carbon, and enhanced nitrogen and strontium are also seen at lower metallicities. For example, ROA $84([\mathrm{Fe} / \mathrm{H}]=-1.35)$ and a star on the MS, S5004811 $([\mathrm{Fe} / \mathrm{H}]=-1.40)$, share similar abundances in carbon and nitrogen. Both have carbon abundances of $[\mathrm{C} / \mathrm{Fe}]=-0.1$, and high nitrogen $\left([\mathrm{N} / \mathrm{Fe}]_{\mathrm{RGB}}=0.8\right.$ and $\left.[\mathrm{N} / \mathrm{Fe}]_{\mathrm{MSTO}}=1.7\right)$. These abundances agree within the errors of measurement. The $s$-process enhancements have somewhat different values, with $[\mathrm{Sr} / \mathrm{Fe}]_{\mathrm{RGB}}=0.5$ and $[\mathrm{Sr} / \mathrm{Fe}]_{\mathrm{MSTO}}=1.3$. ND95 give highresolution abundances for both ROA 84 and 480 which are consistent with these results. For ROA $84[\mathrm{C} / \mathrm{Fe}]=0.05$, $[\mathrm{N} / \mathrm{Fe}]=0.35$, and $[s / \mathrm{Fe}]=0.45$ (where $[s / \mathrm{Fe}]$ is the average values of $[\mathrm{Y} / \mathrm{Fe}]$ and $[\mathrm{Zr} / \mathrm{Fe}]$ ), while for ROA 480 $[\mathrm{C} / \mathrm{Fe}]=-0.65,[\mathrm{~N} / \mathrm{Fe}]=0.80$ and $[s / \mathrm{Fe}]=0.38$.

The upper levels of nitrogen enhancements on the MSTO and RGB are similar, with $[\mathrm{N} / \mathrm{Fe}] \sim 1.8$. This may indicate that enhanced $\mathrm{N}$ abundances are driven more by a phenomenon that is induced in the cluster as a whole. The upper limit on nitrogen enhancements may also indicate that mixing on ascent of the RGB does not occur to the same extent for all stars. If it did, then higher $[\mathrm{N} / \mathrm{Fe}]$ abundances should be measured for objects on the RGB than for those on the MSTO as an increase in nitrogen occurs in the $\mathrm{CN}$ cycle (although given the errors of measurement a difference in the measured abundance between the MSTO and RGB may not be reliable). However, this makes the assumption that the enriched material on the MSTO is uniform throughout the star. In the case where MSTO objects have accreted material onto their surface layers, on ascent of the RGB the convective envelope deepens and dilution of the $\mathrm{N}$ enhanced material occurs. Coupled with this is the increase in $[\mathrm{N} / \mathrm{Fe}]$ due to internal processing and mixing. As the two scenarios (primordial or accretion event) cannot be differentiated due to the complication of mixing on the ascent of the RGB, abundances of elements not effected by this process need to be investigated and compared at the different evolutionary stages. An example of such an element is $\mathrm{Sr}$, and a comparison of the $\mathrm{Sr}$ abundances found for MSTO and RGB stars is described in the following section.

\subsubsection{S2015448 and ROA 276}

As noted in Section 4.4, ROA 276 stood out against all other members of our RGB sample. For our initial adopted metallicity of $[\mathrm{Fe} / \mathrm{H}]=-0.57$, we obtained $[\mathrm{Ba} / \mathrm{Sr}]=-1.2$ from our high-resolution MIKE spectra (see Table 6). (We also found in Section 4.4.3 that this value is quite insensitive to uncertainties in atmospheric parameters.) In contrast, the $[\mathrm{Ba} / \mathrm{Fe}]$ value was found to be equal to or greater than $[\mathrm{Sr} / \mathrm{Fe}]$ for every other star analyzed.

This low $[\mathrm{Ba} / \mathrm{Sr}]$ value for ROA 276 is similar to that found for the MS star S2015448 (Stanford et al. 2006b), which has $[\mathrm{Sr} / \mathrm{Fe}]=1.6,[\mathrm{Ba} / \mathrm{Fe}]<0.6$, and $[\mathrm{Ba} / \mathrm{Sr}]<-1.0$, as may be seen in Table 7 . While the relative frequencies of such large Sr enhancements on the MSTO and RGB are not well determined, and must await further work, the existence of such objects on both the MS and RGB places an important constraint on the origin of the enhancement. Specifically, unless accretion provides a large fraction of the mass of the observed star, a primordial origin of the enhancement is the more likely explanation for the anomaly. That is to say, if the Sr enhancement in S2015448 is confined only to its small convective outer region following accretion of only a small fraction of the star's mass, convective dilution, as described in Equation (2), will lead to a reduced relative abundance, $[\mathrm{Sr} / \mathrm{Fe}]=0.35$, when it reaches the RGB. Conversely (and perhaps more to the point), given the same assumptions, one would conclude that ROA 276 would have had the enormous value, $[\mathrm{Sr} / \mathrm{Fe}] \approx 2.3$, when it was on the MS if it was surface contaminated rather than primordially enhanced. While not outside the realms of possibility, this is unlikely.

This argument can be applied to many of the stars on the RGB with $s$-process enhancements (the carbon and nitrogen abundances are complicated by evolutionary mixing abundance changes) similar to those found at the MSTO (to within errors of measurement). The abundance enhancements seen in these stars are unlikely to have been surface accretion phenomenon unless the mass accreted is very substantial. For example, ROA 279 with $[\mathrm{C} / \mathrm{Fe}]=0.6$ on the RGB would have a carbon abundance of $[\mathrm{C} / \mathrm{Fe}]>2$ on the MS. No stars with such a high carbon abundance ratio were found on the MS.

\section{CONCLUSIONS}

Abundances of $\mathrm{C}, \mathrm{N}$, and the $s$-process elements $\mathrm{Sr}$ and $\mathrm{Ba}$ were determined for a biased sample of 33 RGB stars in $\omega$ Cen. Almost all objects show depletion of carbon, and solar or enhanced nitrogen. The abundances of $\mathrm{Sr}$ and $\mathrm{Ba}$ show enhancement as well. One of the known $\mathrm{CH}$ stars in $\omega$ Cen has been analyzed for the first time for carbon and nitrogen, resulting in $[\mathrm{C} / \mathrm{Fe}]=0.6,[\mathrm{~N} / \mathrm{Fe}]=0.5$, and $[\mathrm{Sr} / \mathrm{Fe}]=[\mathrm{Ba} / \mathrm{Fe}]=0.6$. This star is less enhanced in carbon compared with other $\mathrm{CH}$ stars in the cluster, but still has considerable enhancement in carbon compared with other RGB stars.

The levels of $\mathrm{N}$ enhancement on both the MS and RGB reach similar relative abundances, $[\mathrm{N} / \mathrm{Fe}] \sim 1.8$. This may indicate that mixing on the RGB occurs to different extents for individual stars.

An RGB star with high enhancement of the light $s$-process element $\mathrm{Sr}$ was found, but with little enhancement in $\mathrm{Ba}$. This star is similar to the strongly Sr-enhanced MS object, S2015448 (Stanford et al. 2006b), from which the conclusion is reached that the $\mathrm{Sr}$ enhancement is likely to be primordial in origin, rather than the result of some accretion event.

We thank Don VandenBerg and Leo Girardi for providing unpublished details from their models. Australian access to the Magellan Telescopes was supported through the Major National Research Facilities II program of the Australian Government. We thank the referee for the comments which led to improvements in the manuscript. L.M.S. thanks K. Ward for advice on statistical calculations.

Facilities: ATT (DBS); Magellan:Clay (MIKE)

\section{REFERENCES}

Aoki, W., et al. 2008, ApJ, 678, 1351

Arlandini, C., Käppeler, F., Wisshak, K., Gallino, R., Lugaro, M., Busso, M., \& Straniero, O. 1999, ApJ, 525, 886

Bell, R. A., \& Dickens, R. J. 1974, MNRAS, 166, 89

Briley, M. M., Cohen, J. G., \& Stetson, P. B. 2002, ApJ, 579, 17

Briley, M. M., Harbeck, D., Smith, G. H., \& Grebel, E. K. 2004, AJ, 127, 1588

Brown, J. A., \& Wallerstein, G. 1993, AJ, 106, 133 (BW93)

Calamida, A., et al. 2009, ApJ, 706, 1277

Cannon, R. D., Croke, B. F. W., Bell, R. A., Hesser, J. E., \& Stathakis, R. A. 1998, MNRAS, 298, 601

Cannon, R. D., \& Stobie, R. S. 1973, MNRAS, 162, 207

Charbonnel, C., \& Zahn, J. P. 2007, A\&A, 467, L15

Cottrell, P. L., \& Norris, J. 1978, ApJ, 221, 893 
Cunha, K., Smith, V. V., Suntzeff, N. B., Norris, J. E., Da Costa, G. S., \& Plez, B. 2002, AJ, 124, 379

Da Costa, G. S., Cannon, R., Croke, B., \& Norris, J. E. 2004, Mem. Soc. Astron. Ital., 75, 370

Denissenkov, P. A., \& Pinsonneault, M. 2008, ApJ, 684, 626

Gallino, R., Arlandini, C., Busson, M., Lugaro, M., Travaglio, C., Straniero, O. Chieffi, A., \& Limongi, M. 1998, ApJ, 497, 388

Gratton, R. G. 1982, A\&A, 115, 336

Iben, I. 1965, ApJ, 142, 1447

Johnson, C. I., Pilachowski, C. A., Rich, M., \& Fullbright, M. 2009, ApJ, 698, 2408

Johnson, C. I., Pilachowski, C. A., Simmerer, J., \& Schwenk, D. 2008, ApJ, 681,1505

Kayser, A., Hilker, M., Richtler, T., \& Willemsen, P. G. 2006, A\&A, 458, 777

Kippenhahn, R., Ruschenplatt, G., \& Thomas, H. C. 1980, A\&A, 91, 175

Kurucz, R. 1993, CD-ROM 13, ATLAS9 Stellar Atmosphere Programs and 2 $\mathrm{km} / \mathrm{s}$ Grid (Cambridge: SAO), 13

Langer, G. E., Kraft, R. P., Carbon, D. F., Friel, E., \& Oke, J. B. 1986, PASP, 98,473

Lee, Y.-W., Joo, J.-M., Sohn, Y.-J., Rey, S.-C., Lee, H.-C., \& Walker, A. R. 1999, Nature, 402, 55

Lucatello, S., Beers, T. C., Christleib, N., Barklem, P. S., Rossi, S., Marsteller, B., Sivarani, T., \& Lee, Y. S. 2006, ApJ, 652, L37

Luck, R. E., \& Bond, H. E. 1991, ApJS, 77, 515

Maeder, A., \& Meynet, G. 2006, A\&A, 448, L37

Mayor, M., Duquennoy, A., \& Udry, S. 1996, in ASP Conf. Ser. 90, ed. E. F Milone \& J. C. Mermilliod, The Origins, Evolutions, and Destinies of Binary Stars in Clusters (San Francisco, CA: ASP), 190

McClure, R. D., \& Woodsworth, A. W. 1990, ApJ, 352, 709

Norris, J. E., \& Da Costa, G. S. 1995a, ApJ, 447, 680 (ND95)

Norris, J. E., \& Da Costa, G. S. 1995b, ApJ, 441, L81
Norris, J. E., Freeman, K. C., \& Mighell, K. J. 1996, ApJ, 462, 241

Norris, J. E., Ryan, S. G., \& Beers, T. C. 1997, ApJ, 488, 350

Pancino, E. 2003, PhD thesis, Univ. of Bologna, Italy (PAN03)

Pancino, E., Ferraro, F. R., Bellazzini, M., Piotto, G., \& Zoccali, M. 2000, ApJ, 534, 83

Pancino, E., Pasquini, L., Hill, V., Ferraro, F. R., \& Bellazzini, M. 2002, ApJ, 568, L101

Persson, S. E., Frogel, J. A., Cohen, J. G., Aaronson, M., \& Matthews, K. 1980, ApJ, 235, 452

Piotto, G., et al. 2005, ApJ, 621, 777

Rey, S.-C., Lee, Y.-W., Ree, C. H., Joo, M.-J., \& Sohn, Y.-J. 2004, AJ, 127, 958

Smith, G. H., \& Briley, M. M. 2006, PASP, 118, 740

Smith, V. V., Cunha, K., \& Lambert, D. 1995, AJ, 110, 2827

Smith, V. V., Suntzeff, N., Cunha, K., Gallino, R., Busso, M., Lambert, D., \& Straniero, O. 2000, AJ, 119, 1239

Sollima, A., Ferraro, F. R., Pancino, E., \& Bellazzini, M. 2005, MNRAS, 357, 265

Stancliff, R. J., \& Glebbeek, E. 2008, MNRAS, 389, 1828

Stanford, L. M., Da Costa, G. S., Norris, J. E., \& Cannon, R. D. 2006a, ApJ, 647, 1075

Stanford, L. M., Da Costa, G. S., Norris, J. E., \& Cannon, R. D. 2006b, ApJ, 653, L117

Stanford, L. M., Da Costa, G. S., \& Norris, J. E. 2007, ApJ, 667, 911

Suntzeff, N. B., \& Kraft, R. P. 1996, AJ, 111, 1913

Sweigart, A. V., \& Mengel, J. G. 1979, ApJ, 229, 624

van Leeuwen, F., Le Poole, R. S., Reijns, R. A., Freeman, K. C., \& de Zeeuw, P. T. 2000, A\&A, 360, 472

Ventura, P., D’Antona, F., \& Mazzitelli, I. 2002, A\&A, 393, 215

Villanova, S., et al. 2007, ApJ, 663, 296

Woolley, R. v. d. R., et al. 1966, R. Obs. Ann., 2, 1

Yoshii, Y. 1981, A\&A, 97, 280 